\title{
Extensions of automorphisms of self-similar groups
}

\author{
Francesco Matucci* and Pedro V. Silva \\ Communicated by Dessislava Kochloukova
}

\begin{abstract}
In this work, we study automorphisms of synchronous self-similar groups and the existence of extensions to continuous automorphisms over the closure of these groups with respect to the depth metric. We obtain conditions for the continuity of such extensions, but we also construct examples of groups where such extensions do not exist. We study in detail the case of the lamplighter group $\mathscr{L}_{k}=\mathbb{Z}_{k} \prec \mathbb{Z}$.
\end{abstract}

\section{Introduction}

Let $A$ be a finite alphabet with at least two symbols, and let $A^{\omega}$ be the corresponding Cantor space of all infinite strings. A homeomorphism of $A^{\omega}$ is said to be rational if there exists a transducer (i.e. Mealy machine) that implements the function on infinite strings. There are two different versions of the rational group, depending on the type of transducers we allow. A transducer that outputs exactly one symbol for each symbol that it reads is said to be synchronous, and the group of all homeomorphisms of $A^{\omega}$ determined by synchronous transducers is the synchronous rational group $\wp_{A}$. If we instead allow transducers to output any finite number of symbols at each step, we obtain the asynchronous rational group $\mathcal{R}_{A}$, which includes $\delta_{A}$ as a subgroup.

Subgroups of the synchronous groups $\oiint_{A}$ have received much attention in the literature $[2,3,12]$. In addition to branch groups and self-similar groups such as the Grigorchuk group, several other well-known groups can be represented by synchronous automata, including free groups [15], $\mathrm{GL}_{n}(\mathbb{Z})$ and its subgroups [8], the solvable Baumslag-Solitar groups $\mathrm{BS}(1, m)[6]$, and the lamplighter groups $R \geq \mathbb{Z}$

The first author is a member of the Gruppo Nazionale per le Strutture Algebriche, Geometriche e le loro Applicazioni (GNSAGA) of the Istituto Nazionale di Alta Matematica (INdAM) and gratefully acknowledges the support of the Fundação de Amparo à Pesquisa do Estado de São Paulo (FAPESP Jovens Pesquisadores em Centros Emergentes grant 2016/12196-5), of the Conselho Nacional de Desenvolvimento Científico e Tecnológico (CNPq Bolsa de Produtividade em Pesquisa PQ-2 grant 306614/2016-2) and of the Fundação para a Ciência e a Tecnologia (CEMAT - Ciências FCT projects UIDB/04621/2020 and UIDP/04621/2020). The second author was partially supported by CMUP, which is financed by national funds through FCT - Fundação para a Ciência e a Tecnologia, I.P., under the project with reference UIDB/00144/2020. 
with $R$ a finite ring [13]. In the case of the asynchronous rational group, it has recently been proved that all hyperbolic groups embed into the group $\mathcal{R}_{A}$.

Automorphisms of self-similar groups have been studied in detail before by Lavreniuk and Nekrashevych [11] and Bartholdi and Sidki [4] giving sufficient conditions for automorphisms to be induced by conjugation in $\operatorname{Aut}\left(T_{A}\right)$. In the present paper, we focus on the study of some self-similar groups and their automorphisms, and we investigate their continuity with respect to the depth metric and whether they can be extended to automorphisms of the full group of tree automorphisms of the infinite binary rooted tree. More precisely, since $\delta_{A} \leq \operatorname{Aut}\left(T_{A}\right)$, we define the depth metric on $\operatorname{Aut}\left(T_{A}\right)$ so that, for $\varphi, \psi \in \operatorname{Aut}\left(T_{A}\right)$, we let

$$
d(\varphi, \psi)= \begin{cases}|A|^{-\min \left\{n \in \mathbb{N}|\varphi|_{A^{n}} \neq\left.\psi\right|_{A^{n}}\right\}} & \text { if } \varphi \neq \psi, \\ 0 & \text { if } \varphi=\psi,\end{cases}
$$

where $A^{n}$ is the $n$-th level of the tree $T_{A}$. Given a self-similar group $G \leq \operatorname{Aut}\left(T_{A}\right)$, we will be concerned with its closure, and we will obtain some general results about $\bar{G}$ and about the continuity of extensions of automorphisms of $G$, and show examples with continuous extensions. However, examples with non-continuous extensions exist.

Theorem 1.1. There exists an example of a self-similar group $G \leq \operatorname{Aut}\left(T_{A}\right)$ and an automorphism $\theta: G \rightarrow G$ not admitting a continuous extension to $\bar{G}$.

We specialize our machinery to the particular case of the lamplighter group $\mathscr{L}_{k}=\mathbb{Z}_{k} \prec \mathbb{Z}$ and get the following result.

Theorem 1.2. Every automorphism of $\mathscr{L}_{k}$ admits a continuous extension for the depth metric.

To prove Theorem 1.2, we need to study the structure of the group $\operatorname{Aut}\left(\mathscr{L}_{k}\right)$. Our techniques give alternative proofs of two known results: we find another proof of a theorem of Taback, Stein and Wong [14] describing $\operatorname{Aut}\left(\mathscr{L}_{k}\right)$ and another proof of necessary and sufficient conditions for $\operatorname{Aut}\left(\mathscr{L}_{k}\right)$ to be finitely generated (such conditions were first explored by Bachmuth, Baumslag, Dyer and Mochizuki [1] in the more general setting of $\operatorname{Aut}(G)$ for $G$ a metabelian group).

Our proofs are independent and elementary and focus on the structure of $\mathscr{L}_{k}$ seen as the automaton group of a Cayley machine acting on a Laurent series given by Steinberg and the second author in [13]. Recall that an integer $k \geq 2$ is squarefree if there exists no prime $p \in \mathbb{N}$ such that $p^{2}$ divides $k$. We recover the following result. 
Theorem 1.3 (Bachmuth, Baumslag, Dyer and Mochizuki). Let $k \geq 2$ have positive prime divisors $p_{1}, \ldots, p_{s}$. Then $k$ is squarefree if and only if $\operatorname{Aut}\left(\mathscr{L}_{k}\right)$ is finitely generated.

The paper is organized as follows. In Section 2, we recall all the relevant definitions. In Section 3, we study the depth metric and the closure of self-similar groups in $\operatorname{Aut}\left(T_{A}\right)$ with respect to that metric. In Section 4, we study conditions for the extension of an automorphism to be continuous and produce an example where such an extension is indeed not continuous. In Sections 5 and 6, we focus on the lamplighter group $\mathscr{L}_{k}$ and determine completely its automorphism group proving that it is made of uniformly continuous bijections. Finally, in Section 7, we ask a few questions of interest for future work.

\section{Preliminaries}

Given a finite nonempty set $A$, let $A^{*}$ denote the free monoid on $A$, and let $\varepsilon$ denote the empty word. For a word $u \in A^{*}$, we write $|u|$ to denote its length. Given $u, v \in A^{*}$, we say that $u$ is a prefix of $v$ if $v=u w$ for some $w \in A^{*}$.

We may identify $A^{*}$ with the regular $A$-ary rooted tree $T_{A}$, where $A^{*}$ is the set of nodes, $\varepsilon$ is the root and $u a$ is a child of $u$ for every $u \in A^{*}$ and $a \in A$ (i.e. the parent of $u \in A^{+}=A^{*} \backslash\{\varepsilon\}$ is its maximal proper prefix). An automorphism of $T_{A}$ is then a permutation of $A^{*}$ which preserves length and the prefix relation. We shall often work with a canonical alphabet $A_{n}=\{0,1, \ldots, n-1\}$, and we shall use the notation $T_{n}=T_{A_{n}}$.

Note that $\operatorname{Aut}\left(T_{A}\right)$ is a subgroup of the symmetric group on $A^{*}$. Every map $\varphi \in \operatorname{Aut}\left(T_{A}\right)$ induces an action on the boundary of $T_{A}$, i.e. the set $A^{\omega}$ of all right infinite words $a_{0} a_{1} a_{2} \ldots$. Sometimes, it is more convenient to consider the action of $\operatorname{Aut}\left(T_{A}\right)$ on $A^{\omega}$.

The group $\operatorname{Aut}\left(T_{A}\right)$ is canonically isomorphic to the infinite wreath product $S_{A} \prec S_{A} \prec \cdots$, where $S_{A}$ denotes the symmetric group on $A$. This infinite decomposition involves the so-called local permutations, which we now define.

Given $\varphi \in \operatorname{Aut}\left(T_{A}\right)$ and $u \in A^{*}$, there exists some $\varphi_{u} \in S_{A}$ such that

$$
\text { (ua) } \varphi=(u \varphi)\left(a \varphi_{u}\right) \quad \text { for every } a \in A .
$$

We say that $\varphi_{u}$ is the local permutation induced by $\varphi$ at vertex $u$. We also define the cone automorphism $\varphi_{u} A^{*} \in \operatorname{Aut}\left(T_{A}\right)$ through

$$
(u v) \varphi=(u \varphi)\left(v \varphi_{u A^{*}}\right) \quad\left(v \in A^{*}\right) .
$$

We say that $G \leq \operatorname{Aut}\left(T_{A}\right)$ is a self-similar group if,

$$
\text { for all } \varphi \in G \text { and all } u \in A^{*}, \quad \varphi_{u A^{*}} \in G \text {. }
$$


Finitely generated self-similar groups can be constructed with the help of finite automata/transducers of a particular type. Indeed, we define a Mealy machine to be a structure of the form $\mathcal{M}=(A, Q, \delta, \lambda)$, where

- $A$ is a finite nonempty set (alphabet),

- $Q$ is a finite nonempty set (state set),

- $\delta: Q \times A \rightarrow Q$ is a function (transition function),

- $\lambda: Q \times A \rightarrow A$ is a function (output function).

We say that $\mathcal{M}$ is invertible if the function

$$
\lambda_{q}: A \rightarrow A, \quad a \mapsto(q, a) \lambda
$$

is a permutation for every $q \in Q$. We define an action $Q \times A^{*} \rightarrow Q$ recursively by

- $q \varepsilon=q$,

- $q(u a)=(q u, a) \delta\left(u \in A^{*}, a \in A\right)$.

For every $q \in Q$, we extend $\lambda_{q}$ to a function $\hat{\lambda}_{q}: A^{*} \rightarrow A^{*}$ by setting

- $\varepsilon \widehat{\lambda}_{q}=\varepsilon$,

- $(u a) \widehat{\lambda}_{q}=\left(u \widehat{\lambda}_{q}\right)\left(a \lambda_{q u}\right)\left(u \in A^{*}, a \in A\right)$.

If $\mathcal{M}$ is invertible, then $\widehat{\lambda}_{q}$ is a permutation of $A^{*}$; indeed, $\widehat{\lambda}_{q} \in \operatorname{Aut}\left(T_{A}\right)$. The automaton group generated by $\mathcal{M}$ is the (finitely generated) subgroup of $\operatorname{Aut}\left(T_{A}\right)$ generated by $\left\{\hat{\lambda}_{q} \mid q \in Q\right\}$. It will be denoted by $\mathscr{E}(\mathcal{M})$. Automata groups are precisely the finitely generated self-similar groups.

We present now some examples which will play an important role in the paper.

Example 2.1 (The adding machine). This is the invertible Mealy machine $\mathcal{A}$ depicted by

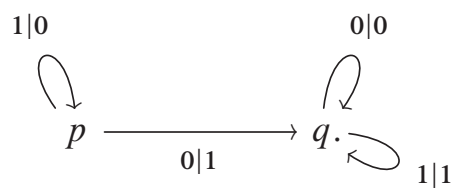

It is well known that $\mathscr{E}(\mathcal{A})=\left\langle\widehat{\lambda}_{p}\right\rangle$ is an infinite cyclic group.

Example 2.2 (The Cayley machine of a finite group). Let $H$ be a finite group. The Cayley machine of $H$, introduced by Krohn and Rhodes in [10], is the invertible Mealy machine $\mathcal{C}_{H}=(H, H, \delta, \lambda)$ defined by

$$
\left(h, h^{\prime}\right) \delta=\left(h, h^{\prime}\right) \lambda=h h^{\prime} \in H \quad\left(h, h^{\prime} \in H\right) .
$$


If $H$ is abelian, Steinberg and the second author proved in [13] that

$$
\mathcal{G}\left(\boldsymbol{C}_{H}\right) \cong H<\mathbb{Z}
$$

the wreath product of $H$ and $\mathbb{Z}$. If $G=C_{2}$ is the group of order 2, we get the Cayley machine

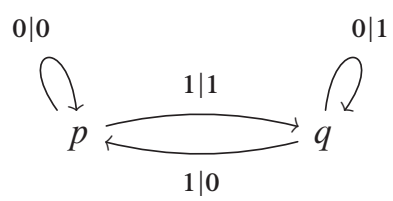

and the lamplighter group $\mathscr{L}_{2}$.

\section{The depth metric}

Let $A$ be a finite nonempty alphabet. We define a metric on $\operatorname{Aut}\left(T_{A}\right)$ as follows. Given $\varphi, \psi \in \operatorname{Aut}\left(T_{A}\right)$, let

$$
d(\varphi, \psi)= \begin{cases}2^{-\min \left\{n \in \mathbb{N}|\varphi|_{A^{n}} \neq\left.\psi\right|_{A^{n}}\right\}} & \text { if } \varphi \neq \psi, \\ 0 & \text { if } \varphi=\psi,\end{cases}
$$

where $A^{n}$ denote the $n$-th level of the tree $T_{A}$. It is immediate that $d$ is indeed an ultrametric on $\operatorname{Aut}\left(T_{A}\right)$, which we call the depth metric. As remarked in [3], the following result follows from Tychonoff's theorem.

Proposition 3.1. The metric space $\left(\operatorname{Aut}\left(T_{A}\right), d\right)$ is compact and therefore complete.

Let $G \leq \operatorname{Aut}\left(T_{A}\right)$. We denote by $\bar{G}$ the topological closure of $G$ in $\left(\operatorname{Aut}\left(T_{A}\right), d\right)$. Note that $\bar{G}$, being a closed subset of a compact space, is itself compact (and therefore complete).

The restrictions of the depth metric to either $G$ or $\bar{G}$ will still be denoted by $d$ and referred to as the depth metric.

It is easy to check that $\bar{G}$ consists precisely of those $\varphi \in \operatorname{Aut}\left(T_{A}\right)$ such that,

$$
\text { for all } n \in \mathbb{N} \text {, there exists } \varphi_{n} \in G \text { such that }\left.\varphi\right|_{A^{n}}=\left.\varphi_{n}\right|_{A^{n}} \text {, }
$$

and is indeed the completion of $(G, d)$. The study of this closure has been introduced for branch groups by Bartholdi, Grigorchuk and Šunić (see [3, Definition 1.18]) under the name tree completion, which we also adopt. Note that $\bar{G}$ is a subgroup of $\operatorname{Aut}\left(T_{A}\right)$. 
Given $n \in \mathbb{N}$, we define the $n$-th level stabilizer of $G \leq \operatorname{Aut}\left(T_{A}\right)$ to be

$$
\operatorname{Stab}_{n}(G)=\left\{\varphi \in G|\varphi|_{A^{n}}=\mathrm{id}\right\} .
$$

It is immediate that $\operatorname{Stab}_{n}(G) \unlhd G$ for every $n$. Moreover, we have a chain

$$
G=\operatorname{Stab}_{0}(G) \supseteq \operatorname{Stab}_{1}(G) \supseteq \operatorname{Stab}_{2}(G) \supseteq \cdots,
$$

where each subgroup has finite index and

$$
\bigcap_{n \in \mathbb{N}} \operatorname{Stab}_{n}(G)=\{\mathrm{id}\}
$$

Therefore, $G$ is residually finite.

Write $G_{n}=G / \operatorname{Stab}_{n}(G)$, and consider the discrete topology on the $G_{n}$. By considering the natural projections $G_{m} \rightarrow G_{n}$ for all $m \geq n$, we obtain a projective system. It is easy to see (see also [3]) that $(\bar{G}, d)$ is the projective limit of the above projective system; hence it is a profinite group (in particular, it is a topological group).

In general, the tree completion is a profinite group [3,9], but it need not coincide with the profinite completion of $G$, when we consider the profinite metric. However, Grigorchuk remarks in [9] that the tree completion coincides with the profinite completion if every finite index subgroup of $G$ contains some $\operatorname{Stab}_{n}(G)$ (that is $G$ satisfies the congruence subgroup property).

Note also that the topology on $G$ induced by $d$ is none other than the topology of pointwise convergence: if we consider $A^{*}$ endowed with the discrete topology, then $\lim _{n \rightarrow+\infty} \varphi_{n}=\varphi$ holds in $G$ if and only if,

$$
\text { for all } u \in A^{*}, \quad \lim _{n \rightarrow+\infty} u \varphi_{n}=u \varphi,
$$

i.e. each sequence $\left(u \varphi_{n}\right)_{n}$ is stationary with limit $u \varphi$.

Lemma 3.2. Let $G \leq \operatorname{Aut}\left(T_{A}\right)$ be self-similar. Then $\bar{G}$ is also a self-similar subgroup of $\operatorname{Aut}\left(T_{A}\right)$.

Proof. We have already noted that $\bar{G} \leq \operatorname{Aut}\left(T_{A}\right)$. Let $\varphi \in \bar{G}$ and $u \in A^{*}$. There exists a sequence $\left(\varphi^{(n)}\right)_{n}$ on $G$ such that $\varphi=\lim _{n \rightarrow+\infty} \varphi^{(n)}$. Let $v \in A^{*}$. It follows easily from continuity that

$$
\begin{aligned}
(u \varphi)\left(v \varphi_{u A^{*}}\right) & =(u v) \varphi=\lim _{n \rightarrow+\infty}(u v) \varphi^{(n)}=\lim _{n \rightarrow+\infty}\left(u \varphi^{(n)}\right)\left(v \varphi_{u A^{*}}^{(n)}\right) \\
& =\left(\lim _{n \rightarrow+\infty} u \varphi^{(n)}\right)\left(\lim _{n \rightarrow+\infty} v \varphi_{u A^{*}}^{(n)}\right)=(u \varphi)\left(\lim _{n \rightarrow+\infty} v \varphi_{u A^{*}}^{(n)}\right),
\end{aligned}
$$


and hence $v \varphi_{u A^{*}}=\lim _{n \rightarrow+\infty} v \varphi_{u A^{*}}^{(n)}$. Since we are dealing with the topology of pointwise convergence and $v$ is arbitrary, we get $\varphi_{u A^{*}}=\lim _{n \rightarrow+\infty} \varphi_{u A^{*}}^{(n)}$. Since $\underline{\varphi}^{(n)} \in G$ and $G$ is self-similar, we have $\varphi_{u A^{*}}^{(n)} \in G$ and so $\varphi_{u A^{*}} \in \bar{G}$. Therefore, $\bar{G}$ is self-similar.

Automata groups, being finitely generated, are always countable. However, the next result shows that their tree completions are countable only in trivial cases.

Lemma 3.3. Let $G \leq \operatorname{Aut}\left(T_{A}\right)$.

(i) If $G$ is finite, then $\bar{G}=G$.

(ii) If $G$ is infinite, then $\bar{G}$ is uncountable.

Proof. (i) True, since every finite subset of a metric space is closed. (ii) True, since $\bar{G}$ is an infinite profinite group.

We shall prove that $\bar{G}=\operatorname{Aut}\left(T_{A}\right)$ for an automaton group $G$ only in trivial cases. But we start with the following lemma, where the rank of a finitely generated group $G$ denotes the minimum cardinality of a generating set of $G$.

We shall denote by $T_{A}^{(n)}$ the (rooted) subtree of $T_{A}$ induced by the nodes $A^{\leq n}$. It is immediate to see that $\operatorname{Aut}\left(T_{A}^{(n)}\right) \cong\left(\operatorname{Aut}\left(T_{A}\right)\right) / \operatorname{Stab}_{n}\left(\operatorname{Aut}\left(T_{A}\right)\right)$. The following result is well known (see for example Brunner and Sidki [7, Section 2]).

Lemma 3.4. Let $A$ be a finite alphabet with $|A| \geq 2$, and let $n \geq 1$. Then

$$
\operatorname{rank}\left(\operatorname{Aut}\left(T_{A}^{(n)}\right)\right) \geq n .
$$

We can now prove the following.

Proposition 3.5. Let $A$ be a finite alphabet with $|A| \geq 2$, and let $G \leq \operatorname{Aut}\left(T_{A}\right)$ be finitely generated. Then $\bar{G}<\operatorname{Aut}\left(T_{A}\right)$.

Proof. Since $G$ is finitely generated, it follows that $\bar{G}$ is topologically finitely generated. However, it is well known that $\operatorname{Aut}\left(T_{A}\right)$ is not topologically finitely generated.

We also offer a short proof not involving profinite groups. Suppose that we have $\bar{G}=\operatorname{Aut}\left(T_{A}\right)$. It follows easily from the definition of closure that $G$ and $\bar{G}$ induce the same automorphisms of $T_{A}^{(n)}$ for every $n \in \mathbb{N}$. Hence every $\psi \in \operatorname{Aut}\left(T_{A}^{(n)}\right)$ is a restriction of some $\varphi \in G$. It follows that $\operatorname{Aut}\left(T_{A}^{(n)}\right) \cong G / \operatorname{Stab}_{n}(G)$ for every $n \in \mathbb{N}$. 
By Lemma 3.4, $\operatorname{rank}\left(\operatorname{Aut}\left(T_{A}^{(k+1)}\right)\right) \geq k+1$ for every positive integer $k$. If we let $m=\operatorname{rank}(G)$, then we notice that

$$
m+1 \leq \operatorname{rank}\left(\operatorname{Aut}\left(T_{A}^{(m+1)}\right)\right)=\operatorname{rank}\left(G / \operatorname{Stab}_{m+1}(G)\right) \leq \operatorname{rank}(G)=m,
$$

so we get a contradiction. Therefore, $\bar{G}<\operatorname{Aut}\left(T_{A}\right)$.

In particular, we have $\bar{G}<\operatorname{Aut}\left(T_{A}\right)$ for every automaton group over an alphabet with at least two letters.

\section{Automorphisms of an automaton group}

\subsection{General results}

It is a natural question to inquire which endomorphisms of an automaton group admit a continuous extension to $(\bar{G}, d)$. General topology yields the following result.

Lemma 4.1. Let $G \leq \operatorname{Aut}\left(T_{A}\right)$, and let $\theta: G \rightarrow G$ be a function. Then the following conditions are equivalent:

(i) $\theta$ admits a continuous extension to $(\bar{G}, d)$;

(ii) $\theta$ is uniformly continuous in $(G, d)$.

Proof. (i) $\Rightarrow$ (ii). Since $\left(\operatorname{Aut}\left(T_{A}\right), d\right)$ is compact by Proposition 3.1 and $\bar{G}$ is a closed subset of $\operatorname{Aut}\left(T_{A}\right)$, it follows that $(\bar{G}, d)$ is itself compact (and therefore complete - it is the completion of $(G, d)$ ). Hence a continuous extension of $\theta$ to $\bar{G}$ is thus a continuous map over a compact set, so it is necessarily uniformly continuous with respect to $d$, and its restriction is $\theta$.

(ii) $\Rightarrow$ (i). Every uniformly continuous transformation of $(G, d)$ admits a continuous extension to its completion, which is precisely $(\bar{G}, d)$.

Note that, if there is a continuous extension of the function $\theta: G \rightarrow G$ to its closure $\bar{G}$, it is unique. If an automorphism admits a continuous extension, we show that the latter is also an automorphism.

Proposition 4.2. Let $G \leq \operatorname{Aut}\left(T_{A}\right)$, and let $\theta \in \operatorname{Aut}(G)$ be such that both $\theta$ and $\theta^{-1}$ are uniformly continuous. Then its continuous extension $\bar{\theta}: \bar{G} \rightarrow \bar{G}$ is also an automorphism.

Proof. Let $\varphi, \psi \in \bar{G}$. Then there exist sequences $\left(\varphi^{(n)}\right)_{n}$ and $\left(\psi^{(n)}\right)_{n}$ on $G$ such that $\varphi=\lim _{n \rightarrow+\infty} \varphi^{(n)}$ and $\psi=\lim _{n \rightarrow+\infty} \psi^{(n)}$. By continuity, we get

$$
(\varphi \psi) \bar{\theta}=\left(\left(\lim _{n \rightarrow+\infty} \varphi^{(n)}\right)\left(\lim _{n \rightarrow+\infty} \psi^{(n)}\right)\right) \bar{\theta}=\left(\lim _{n \rightarrow+\infty}\left(\varphi^{(n)} \psi^{(n)}\right)\right) \bar{\theta}
$$




$$
\begin{aligned}
& =\lim _{n \rightarrow+\infty}\left(\left(\varphi^{(n)} \psi^{(n)}\right) \theta\right)=\lim _{n \rightarrow+\infty}\left(\left(\varphi^{(n)} \theta\right)\left(\psi^{(n)} \theta\right)\right) \\
& =\left(\lim _{n \rightarrow+\infty}\left(\varphi^{(n)} \theta\right)\right)\left(\lim _{n \rightarrow+\infty}\left(\psi^{(n)} \theta\right)\right) \\
& =\left(\lim _{n \rightarrow+\infty} \varphi^{(n)}\right) \bar{\theta}\left(\lim _{n \rightarrow+\infty} \psi^{(n)}\right) \bar{\theta}=(\varphi \bar{\theta})(\psi \bar{\theta}),
\end{aligned}
$$

where the second equality follows from the fact that $\varphi, \psi$ respectively eventually coincide with $\varphi^{(n)}, \psi^{(n)}$ for $n$ large enough, and so $\varphi \psi$ eventually coincides with $\varphi^{(n)} \psi^{(n)}$ for $n$ large enough. Therefore, $\bar{\theta}$ is a group homomorphism. Since $\overline{\theta^{-1}}$ is also a group homomorphism, it is easy to see that $\bar{\theta}$ and $\overline{\theta^{-1}}$ are mutually inverse. Therefore, $\bar{\theta} \in \operatorname{Aut}(\bar{G})$.

We can relate uniform continuity to stabilizers in the case of automorphisms.

Proposition 4.3. Let $G \leq \operatorname{Aut}\left(T_{A}\right)$, and let $\theta: G \rightarrow G$ be an automorphism. Then the following conditions are equivalent:

(i) $\theta$ is uniformly continuous in $(G, d)$;

(ii) for all $m \in \mathbb{N}$, there exists $n \in \mathbb{N}$ such that $\left(\operatorname{Stab}_{n}(G)\right) \theta \subseteq \operatorname{Stab}_{m}(G)$.

Proof. In fact, condition (i) is equivalent to the following equivalent statements:

for all $m \in \mathbb{N}$, there exists $n \in \mathbb{N}$ such that, for all $\varphi, \psi \in G$,

$$
d(\varphi, \psi)<2^{-n} \Longrightarrow d(\varphi \theta, \psi \theta)<2^{-m}
$$

$\Longleftrightarrow$ for all $m \in \mathbb{N}$, there exists $n \in \mathbb{N}$ such that, for all $\varphi, \psi \in G$,

$$
\left.\varphi\right|_{A^{n}}=\left.\left.\psi\right|_{A^{n}} \Longrightarrow(\varphi \theta)\right|_{A^{m}}=\left.(\psi \theta)\right|_{A^{m}}
$$

$\Longleftrightarrow$ for all $m \in \mathbb{N}$, there exists $n \in \mathbb{N}$ such that, for all $\varphi, \psi \in G$,

$$
\psi^{-1} \varphi \in \operatorname{Stab}_{n}(G) \Longrightarrow\left(\psi^{-1} \varphi\right) \theta \in \operatorname{Stab}_{m}(G)
$$

$\Longleftrightarrow$ for all $m \in \mathbb{N}$, there exists $n \in \mathbb{N}$ such that

$$
\left(\operatorname{Stab}_{n}(G)\right) \theta \subseteq \operatorname{Stab}_{m}(G)
$$

Corollary 4.4. Let $G \leq \operatorname{Aut}\left(T_{A}\right)$, and let $\theta: G \rightarrow G$ be an inner automorphism. Then $\theta$ is uniformly continuous in $(G, d)$.

Proof. By Proposition 4.3, since $\left(\operatorname{Stab}_{n}(G)\right) \theta=\operatorname{Stab}_{n}(G)$ for every inner automorphism $\theta$ of $G$.

Recall that a subgroup $H$ of a group $G$ is characteristic if $\varphi(H) \leq H$ for all $\varphi \in \operatorname{Aut}(G)$. 
Corollary 4.5. Let $G \leq \operatorname{Aut}\left(T_{A}\right)$ be such that $\operatorname{Stab}_{n}(G)$ is characteristic for every $n \in \mathbb{N}$. Then every automorphism of $G$ is uniformly continuous in $(G, d)$.

It is natural to ask whether or not an automorphism of a self-similar group is itself a restriction of a continuous automorphism of $\operatorname{Aut}\left(T_{A}\right)$. The following remark is a necessary condition tying the existence of extensions to $\operatorname{Aut}(\bar{G})$ to extensions to $\operatorname{Aut}\left(\operatorname{Aut}\left(T_{A}\right)\right)$. In Subsections 4.3 and 4.4, we see examples where extensions to $\operatorname{Aut}\left(\operatorname{Aut}\left(T_{A}\right)\right)$ may or may not exist.

Remark 4.6. Given a self-similar group $G \leq \operatorname{Aut}\left(T_{A}\right)$ and $\theta \in \operatorname{Aut}(G)$, we observe that, if there exists $\varphi \in \operatorname{Aut}\left(\operatorname{Aut}\left(T_{A}\right)\right)$ continuous such that $\left.\varphi\right|_{G}=\theta$, the restriction $\left.\varphi\right|_{G}$ is indeed an element of $\operatorname{Aut}(\bar{G})$ and a continuous extension of $\theta$.

\subsection{Aleshin's automaton and automorphisms}

The following example shows that condition (ii) of Proposition 4.3 does not hold for every automaton group. Let $G$ be the automaton group generated by Aleshin's automaton

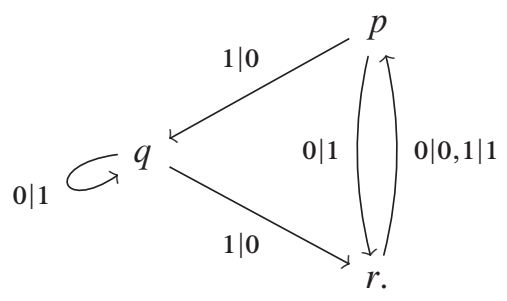

We claim that $\operatorname{Stab}_{2}(G)$ is not characteristic. Indeed, Vorobets and Vorobets [15] proved that $G$ is the free group on $\left\{\widehat{\lambda}_{p}, \widehat{\lambda}_{q}, \widehat{\lambda}_{r}\right\}$; hence $\widehat{\lambda}_{p} \mapsto \widehat{\lambda}_{r} \widehat{\lambda}_{p}, \widehat{\lambda}_{q} \mapsto \widehat{\lambda}_{q}$, $\hat{\lambda}_{r} \mapsto \hat{\lambda}_{r}$ defines an elementary Nielsen automorphism $\theta$ of $G$. The action of the generators of $G$ at depth 2 is described by the diagram

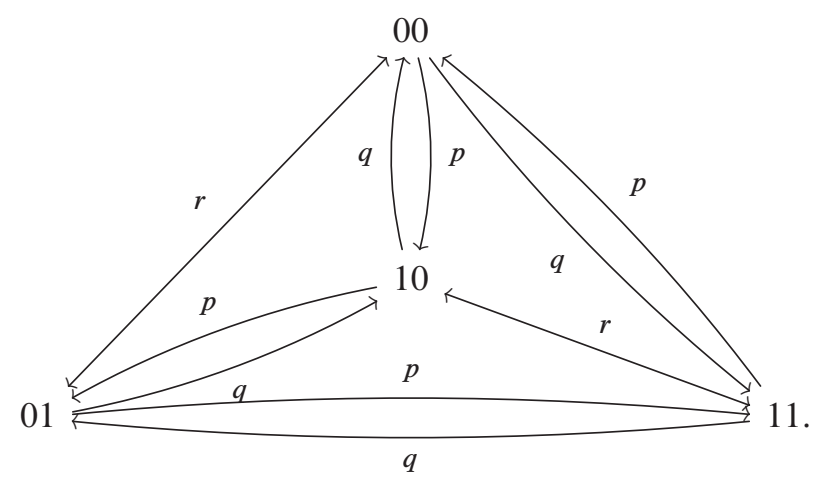


It follows easily that $\widehat{\lambda}_{p} \widehat{\lambda}_{q} \in \operatorname{Stab}_{2}(G)$ but $\left(\widehat{\lambda}_{p} \widehat{\lambda}_{q}\right) \theta=\widehat{\lambda}_{r} \widehat{\lambda}_{p} \widehat{\lambda}_{q} \notin \operatorname{Stab}_{2}(G)$. Therefore, $\operatorname{Stab}_{2}(G)$ is not characteristic.

\subsection{The adding machine and its automorphisms}

We consider now the adding machine to illustrate some of the problems already introduced and some others we intend to propose. Write $\varphi=\widehat{\lambda}_{p}$ in the notation of Example 2.1. Since $\mathcal{A}$ generates the infinite cyclic group $\mathscr{E}(\mathcal{A})=\langle\varphi\rangle$, there exist only two automorphisms of $\mathscr{G}(\mathcal{A})$ : the identity and the automorphism $\theta$ defined by

$$
\varphi^{n} \mapsto \varphi^{-n} \quad(n \in \mathbb{Z}) .
$$

The adding machine takes its name from the fact that it reproduces addition of integers in binary form in the following sense: if $u \in A_{2}^{m}$, then $u \varphi^{n} \in A_{2}^{m}$ is the unique integer $v$ in binary form such that $\widetilde{v}$ is congruent to $\widetilde{u}+n$ modulo $2^{m}$, where $\widetilde{w}$ is the word $w$ read in reverse order. It follows that

$$
\operatorname{Stab}_{m}(\mathscr{E}(\mathcal{A}))=\left\langle\varphi^{2^{m}}\right\rangle
$$

for every $m \in \mathbb{N}$. But then $\operatorname{Stab}_{m}(\mathscr{E}(\mathcal{A}))$ is a characteristic subgroup of $\mathscr{E}(\mathcal{A})$ for every $m \in \mathbb{N}$, and so every automorphism of $\mathscr{G}(\mathcal{A})$ admits a continuous extension to $\bar{G}$ by Lemma 4.1 and Corollary 4.5 .

We also claim that every automorphism of $\mathscr{E}(\mathcal{A})$ can be actually extended to an automorphism of $\operatorname{Aut}\left(T_{2}\right)$. This is trivial for the identity automorphism, so we only have to consider $\theta$. We show that $\theta$ is the restriction to $\mathscr{E}(\mathcal{A})$ of the mirror image automorphism $\mu$ of $\operatorname{Aut}\left(T_{2}\right)$.

Each $\varphi \in \operatorname{Aut}\left(T_{2}\right)$ is fully determined by the local permutations $\varphi_{u}\left(u \in A_{2}^{*}\right)$. Let $\sigma \in \operatorname{Aut}\left(A_{2}^{*}\right)$ swap 0 and 1 . We define $\varphi \mu \in \operatorname{Aut}\left(T_{2}\right)$ by

$$
(\varphi \mu)_{u}=\varphi_{u \sigma} \quad\left(u \in A_{2}^{*}\right) .
$$

We must prove that $\mu$ is an automorphism of $\operatorname{Aut}\left(T_{2}\right)$. We start by showing that

$$
u(\varphi \mu) \sigma=u \sigma \varphi
$$

for all $\varphi \in \operatorname{Aut}\left(T_{2}\right)$ and $u \in A_{2}^{*}$. We use induction on $|u|$. The claim holds trivially for $u=\varepsilon$; hence we assume that $u=v a$ with $v \in A_{2}^{*}$ and $a \in A_{2}$, and that (4.2) holds for $v$. We have

$$
u(\varphi \mu) \sigma=(v a)(\varphi \mu) \sigma=\left((v(\varphi \mu))\left(a(\varphi \mu)_{v}\right)\right) \sigma=(v(\varphi \mu) \sigma)\left(a \varphi_{v \sigma} \sigma\right) .
$$

By the induction hypothesis, we get

$$
u(\varphi \mu) \sigma=(v \sigma \varphi)\left(a \sigma \varphi_{v \sigma}\right)=((v \sigma)(a \sigma)) \varphi=(v a) \sigma \varphi=u \sigma \varphi,
$$

where $\varphi_{v \sigma} \sigma=\sigma \varphi_{v \sigma}$ since $S_{A_{2}}$ is abelian. Therefore, (4.2) holds. 
Now let $\varphi, \psi \in \operatorname{Aut}\left(T_{2}\right)$. For every $u \in A_{2}^{*}$, (4.1) and (4.2) yield

$$
\begin{aligned}
((\varphi \psi) \mu)_{u} & =(\varphi \psi)_{u \sigma}=\varphi_{u \sigma} \psi_{u \sigma \varphi}=\varphi_{u \sigma} \psi_{u(\varphi \mu) \sigma} \\
& =(\varphi \mu)_{u}(\psi \mu)_{u(\varphi \mu)}=((\varphi \mu)(\psi \mu))_{u} ;
\end{aligned}
$$

hence $(\varphi \psi) \mu=(\varphi \mu)(\psi \mu)$, and so $\mu$ is a group homomorphism. Since $\mu$ is clearly bijective, it is an automorphism of $\operatorname{Aut}\left(T_{2}\right)$.

Finally, we show that $\theta=\left.\mu\right|_{\mathscr{E}(\mathcal{A})}$. Writing $\varphi=\widehat{\lambda}_{p}$, it suffices to show that $\varphi \theta=\varphi \mu$. Let $u \in A_{2}^{*}$. We must show that

$$
(\varphi \theta)_{u}=(\varphi \mu)_{u} .
$$

Note that (4.1) and the fact that the local functions are in $S_{A_{2}}$ imply

$$
\left(\varphi^{-1}\right)_{u}=\varphi_{u \varphi^{-1}}
$$

hence

$$
(\varphi \theta)_{u}=\left(\varphi^{-1}\right)_{u}=\varphi_{u \varphi^{-1}}
$$

On the other hand,

$$
(\varphi \mu)_{u}=\varphi_{u \sigma} .
$$

It follows easily from the construction of the adding machine that

$$
\varphi_{v}= \begin{cases}\text { id } & \text { if } v \in 0 A_{2}^{*}, \\ (01) & \text { otherwise. }\end{cases}
$$

Now $u \sigma \in 0 A_{2}^{*}$ if and only if $u \in 1 A_{2}^{*}$ if and only if $u \varphi^{-1} \in 0 A_{2}^{*}$; hence we have $\varphi_{u \sigma}=\varphi_{u \varphi^{-1}}$, and so (4.3) follows from (4.4) and (4.5). Therefore, $\theta=\left.\mu\right|_{\mathscr{E}(\mathcal{A})}$, and so every automorphism of $\mathscr{E}(\mathcal{A})$ is a restriction of some automorphism of $\operatorname{Aut}\left(T_{2}\right)$.

For $\theta \in \operatorname{Aut}(\mathscr{G}(\mathcal{A}))$, we can also compute the subgroup of fixed points

$$
\operatorname{Fix}(\theta)=\{\psi \in \mathscr{E}(\mathcal{A}) \mid \psi \theta=\psi\} .
$$

Since $\varphi^{n} \theta=\varphi^{-n}$, we have $\operatorname{Fix}(\theta)=\{\operatorname{id}\}$, hence finitely generated. We shall see in the next section that this is not always the case.

\subsection{A non-uniformly continuous automorphism}

In this subsection, we prove Theorem 1.1 by describing a construction to show that a direct product of automata groups is itself an automaton group. The direct product result is well known (for example, see the construction mentioned in the survey [5, immediately after Theorem 2.2] and Remark 4.8 below). However, to 
the best of our knowledge, the construction described in the proof below is new and potentially of independent interest, and we will use it to show an application afterwards.

Lemma 4.7. If $G$ and $H$ are automata groups, $G \times H$ is an automaton group too.

Proof. We can assume that $G=\mathscr{G}(\mathcal{A}) \leq \operatorname{Aut}\left(T_{A}\right)$ and $H=\mathscr{G}(\mathscr{B}) \leq \operatorname{Aut}\left(T_{B}\right)$ are automata groups, where the automata $\mathcal{A}$ and $\mathscr{B}$ are such that the alphabets $A$ and $B$ are disjoint. We now construct two new automata $\mathcal{A}^{\prime}$ and $\mathscr{B}^{\prime}$ on the same alphabet $C=A \cup B$. The automaton $\mathcal{A}^{\prime}$ has the same states of $\mathcal{A}$ and has the same transition and output function on the elements of $A$, while $(b, q) \delta_{\mathcal{A}^{\prime}}=q$ and $(b, q) \lambda_{\mathcal{A}^{\prime}}=b$ for all states $q \in \mathcal{A}^{\prime}$ and $b \in B$. In a similar fashion, $\mathscr{B}^{\prime}$ has the same states of $\mathscr{B}$ and has the same transition and output function on the elements of $B$, while $(a, q) \delta_{\mathcal{B}^{\prime}}=q$ and $(a, q) \lambda_{\mathscr{B}^{\prime}}=a$ for all states $q \in \mathscr{B}^{\prime}$ and $a \in A$. Hence the transition and output functions behave as shown in the picture below:
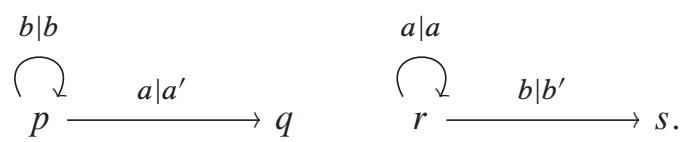

Let $\mathcal{C}$ be the automaton given by the disjoint union of $\mathcal{A}^{\prime}$ and $\mathscr{B}^{\prime}$. Observe that $\mathscr{H}\left(\mathcal{A}^{\prime}\right)=\left\langle Q_{\mathscr{A}}\right\rangle$ and $\mathscr{E}\left(\mathscr{B}^{\prime}\right)=\left\langle Q_{\mathcal{B}}\right\rangle$, where $Q_{\mathcal{A}}$ is the set of rational maps on the alphabet $A$ given by taking each state of $\mathcal{A}$ as the initial state of the automaton. We also define $Q_{\mathcal{B}}$ accordingly. By construction of $\mathcal{A}^{\prime}$ and $\mathscr{B}^{\prime}$, it is clear that $g h=h g$ for all $g \in G$ and $h \in H$ and that $G \cap H=\{1\}$; therefore, we have that $\mathscr{E}(\mathcal{C}) \cong G \times H$, and so $G \times H$ is an automaton group.

Remark 4.8. The direct product construction mentioned in [5] constructs the direct product of automata groups as an automaton group by taking the direct product of the alphabets and produces a connected automaton for $G \times H$, while the construction above keeps previously existing connected components disjoint from each other.

We will now use Proposition 4.3 and Lemma 4.7 to construct an example of a non-uniformly continuous automorphism of an automaton group. Consider the following automaton $\mathcal{A}$ obtained by taking the adding machine automaton and, at each state, replacing the local permutation with its inverse (this construction is what is usually referred to as the inverse automaton):

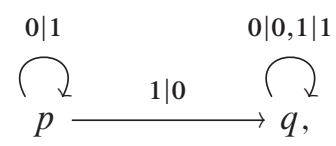


and let $p$ be the rational function arising from the automaton with initial state $p$. By construction,

$$
1^{\omega} \rightarrow^{p} 01^{\omega} \rightarrow^{p} 101^{\omega} \rightarrow^{p} 001^{\omega} \rightarrow^{p} 1101^{\omega} \rightarrow^{p} \cdots,
$$

and so, since each image through $p$ will have a tail given by $01^{\omega}$, it follows that $p$ has infinite order and $\mathscr{E}(\mathcal{A})=\langle p\rangle \cong \mathbb{Z}$. Consider the following automaton $\mathscr{B}$ :

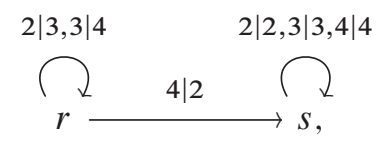

and let $r$ be the rational function coming from the automaton with initial state $r$. By construction,

$$
4^{\omega} \rightarrow^{r} 24^{\omega} \rightarrow^{r} 324^{\omega} \rightarrow^{r} 4324^{\omega} \rightarrow^{r} 2324^{\omega} \rightarrow^{r} 34324^{\omega} \rightarrow^{r} \cdots,
$$

and so, since each image through $p$ will have a tail given by $24^{\omega}, r$ has infinite order and $\mathscr{E}(\mathscr{B})=\langle r\rangle \cong \mathbb{Z}$. If we build the automaton $\mathcal{C}$ as in the proof of Lemma 4.7, then $\mathscr{E}(\mathcal{C})=\langle p, r\rangle \cong \mathbb{Z}^{2}$. Let $\theta \in \operatorname{Aut}(\mathscr{E}(\mathcal{C}))$ be the automorphism swapping $p$ with $r$. We argue by contradiction that $\theta$ is not uniformly continuous. By Proposition 4.3, there exists a positive integer $n$ such that

$$
\left(\operatorname{Stab}_{n}(\mathscr{E}(\mathcal{C}))\right) \theta \subseteq \operatorname{Stab}_{1}(\mathcal{E}(\mathcal{C})) .
$$

Observe that, for every $g \in \operatorname{Aut}\left(T_{2}\right)$, we have $g^{2^{n}}=$ id at level $n$. In fact, by induction, if $g^{2^{n-1}}$ fixes level $n-1$ pointwise, then even if it swapped some of the children of level $n-1$, we have that $\left(g^{2^{n-1}}\right)^{2}=g^{2^{n}}$ would fix all such children. Hence, in particular, $p^{2^{n}} \in \operatorname{Stab}_{n}(\mathcal{E}(\mathcal{E}))$, and therefore,

$$
r^{2^{m}}=\left(p^{2^{m}}\right) \theta \in \operatorname{Stab}_{1}(\mathcal{E}(\mathcal{C})) .
$$

However, since $r$ acts as the 3-cycle (2 34 ) at level 1, it follows that $(234)^{2^{m}}=\mathrm{id}$, and so $3=\left|\left(\begin{array}{ll}2 & 3\end{array}\right)\right|$ divides $2^{m}$, which is a contradiction and implies that $\theta$ is not uniformly continuous.

Another way to rephrase the previous counterexample is to consider the profinite group $\mathbb{Z}[2] \oplus \mathbb{Z}[3]$ seen as the profinite closure of its dense subgroup $\mathbb{Z} \oplus \mathbb{Z}$ and where the symbol $\mathbb{Z}[p]$ denotes the group of $p$-adic integers. If we consider the automorphism of $\mathbb{Z} \oplus \mathbb{Z}$ that swaps the two generators of the infinite cyclic groups $\mathbb{Z}$, then this automorphism does not extend to a continuous automorphism of $\mathbb{Z}[2] \oplus \mathbb{Z}[3]$. Thus, Theorem 1.1 is proved. 


\section{Cayley machines of finite abelian groups}

In the current and following section, we work on proving Theorem 1.2. We follow the notation from [13]. Let $(H,+)$ be a finite nontrivial abelian group in additive notation. We consider the action of $\mathcal{E}\left(\mathcal{C}_{H}\right)$ on the boundary of the tree $T_{H}$. For all $h, x_{0}, x_{1}, \ldots \in H$, we have

$$
\left(x_{0}, x_{1}, x_{2}, \ldots\right) \widehat{\lambda}_{h}=\left(h+x_{0}, h+x_{0}+x_{1}, h+x_{0}+x_{1}+x_{2}, \ldots\right) .
$$

Let $\xi=\widehat{\lambda}_{0}$. For every $h \in H$, let $\alpha_{h}=\widehat{\lambda}_{h} \xi^{-1}$. Since $\mathcal{E}\left(\boldsymbol{C}_{H}\right)=\left\langle\widehat{\lambda}_{h} \mid h \in H\right\rangle$, we also have

$$
\mathscr{I}\left(\boldsymbol{C}_{H}\right)=\left\langle\xi, \alpha_{h} \mid h \in H\right\rangle \text {. }
$$

Note that

$$
\begin{aligned}
\left(x_{0}, x_{1}, x_{2}, \ldots\right) \xi & =\left(x_{0}, x_{0}+x_{1}, x_{0}+x_{1}+x_{2}, \ldots\right), \\
\left(x_{0}, x_{1}, x_{2}, \ldots\right) \xi^{-1} & =\left(x_{0},-x_{0}+x_{1},-x_{1}+x_{2}, \ldots\right), \\
\left(x_{0}, x_{1}, x_{2}, \ldots\right) \alpha_{h} & =\left(h+x_{0}, x_{1}, x_{2}, \ldots\right)
\end{aligned}
$$

We can identify $\left(x_{0}, x_{1}, x_{2}, \ldots\right) \in H^{\omega}$ with the formal power series

$$
X=\sum_{n=0}^{\infty} x_{n} t^{n} \in H[[t]] .
$$

Then we have

$$
X \xi^{n}=X(1-t)^{-n}, \quad X \xi^{n} \alpha_{h} \xi^{-n}=X+h(1-t)^{n}
$$

for all $X \in H[[t]], n \in \mathbb{Z}$ and where of course we mean

$$
(1-t)^{-1}=\sum_{i=0}^{\infty} t^{i} .
$$

By [13], every $\varphi \in \mathscr{S}\left(\boldsymbol{C}_{H}\right)$ admits a unique factorization of the form

$$
\varphi=\left(\xi^{n_{1}} \alpha_{h_{1}} \xi^{-n_{1}}\right) \ldots\left(\xi^{n_{k}} \alpha_{h_{k}} \xi^{-n_{k}}\right) \xi^{m},
$$

with $k \in \mathbb{N} ; n_{i}, m \in \mathbb{Z} ; n_{1}<\cdots<n_{k} ; h_{i} \in H \backslash\{1\}$. By a slight abuse of notation, we identify elements $h \in H$ with the maps $\alpha_{h}$, and thus we observe that

$$
\left\langle H, \xi \mid\left[\xi^{m} h \xi^{-m}, \xi^{n} h^{\prime} \xi^{-n}\right]\left(h, h^{\prime} \in H ; m, n \in \mathbb{Z}\right)\right\rangle
$$

constitutes a relative presentation of $\mathcal{E}\left(\boldsymbol{C}_{H}\right) \cong H<\mathbb{Z}$. 


\subsection{Endomorphisms of the lamplighter group}

We now fix $k \geq 2$ and consider the lamplighter group $\mathscr{L}_{k}=\mathscr{E}\left(\mathcal{C}_{C_{k}}\right)$. We identify $C_{k}$ with $\mathbb{Z}_{k}$, the integers modulo $k$ under addition. We denote by $\mathbb{Z}_{k}^{*}$ the subgroup of units of $\left(\mathbb{Z}_{k}, \cdot\right)$ (i.e. the integers modulo $k$ coprime with $k$ ). Writing $\alpha=\alpha_{1}$, we get a presentation of $\mathscr{L}_{k}$ of the form

$$
\left\langle\alpha, \xi \mid \alpha^{k},\left[\xi^{m} \alpha \xi^{-m}, \xi^{n} \alpha \xi^{-n}\right](m, n \in \mathbb{Z})\right\rangle .
$$

Note that $\xi^{m_{0}} \alpha^{n_{1}} \xi^{m_{1}} \ldots \alpha^{n_{k}} \xi^{m_{k}}$ has finite order if and only if

$$
m_{0}+m_{1}+\cdots+m_{k}=0 .
$$

Let Fin $\left(\mathscr{L}_{k}\right)$ denote the subset of all elements of $\mathscr{L}_{k}$ of finite order. Then Fin $\left(\mathscr{L}_{k}\right)$ is an abelian normal subgroup of $\mathscr{L}_{k}$, and $\mathscr{L}_{k} / \operatorname{Fin}\left(\mathscr{L}_{k}\right)$ is infinite cyclic. Moreover, $\operatorname{Fin}\left(\mathscr{L}_{k}\right)$ is a direct sum of countably many cyclic groups of order $k$.

For every $m \in \mathbb{Z}$, we write $\beta_{m}=\xi^{m} \alpha \xi^{-m}$. It follows easily from (5.1) that each $x \in \mathscr{L}_{k}$ can be written uniquely in the form

$$
x=\left(\prod_{n \in \mathbb{Z}} \beta_{n}^{i_{n}}\right) \xi^{r},
$$

where $r \in \mathbb{Z}$ and $\left(i_{n}\right)_{n}$ is a function $\mathbb{Z} \rightarrow \mathbb{Z}_{k}$ with finite support (i.e. only finitely many terms $i_{n}$ are nonzero). Moreover, $x \in \operatorname{Fin}\left(\mathscr{L}_{k}\right)$ if and only if $r=0$.

How can we characterize the endomorphisms $\varphi$ of $\mathscr{L}_{k}$ ? Since $\mathscr{L}_{k}$ is generated by $\alpha$ and $\xi, \varphi$ is fully determined by the images of $\alpha$ and $\xi$. It follows easily from (5.1) that $\alpha \varphi$ can be any element $y \in \operatorname{Fin}\left(\mathscr{L}_{k}\right)$ and $\xi \varphi$ can be any element $z \in \mathscr{L}_{k}$ : indeed, the equalities $y^{k}=1$ and $\left[z^{m} y z^{-m}, z^{n} y z^{-n}\right]=1$ follow from the fact that $z^{n} y z^{-n} \in \operatorname{Fin}\left(\mathscr{L}_{k}\right)$ for every $n$, and $\operatorname{Fin}\left(\mathscr{L}_{k}\right)$ is an abelian group of exponent $k$.

Given functions $\left(i_{n}\right)_{n}$ and $\left(j_{n}\right)_{n}$ from $\mathbb{Z}$ to $\mathbb{Z}_{k}$ with finite support and $r \in \mathbb{Z}$, and in view of the normal form defined before, the correspondence

$$
\alpha \mapsto \prod_{n \in \mathbb{Z}} \beta_{n}^{i_{n}}, \quad \xi \mapsto\left(\prod_{n \in \mathbb{Z}} \beta_{n}^{j_{n}}\right) \xi^{r}
$$

induces an endomorphism of $\mathscr{L}_{k}$, which we denote by $\varphi_{\left(i_{n}\right)_{n},\left(j_{n}\right)_{n}, r}$. Conversely, every endomorphism can be written uniquely in this form. We next show that

$$
\beta_{m} \varphi_{\left(i_{n}\right)_{n},\left(j_{n}\right)_{n}, r}=\prod_{n \in \mathbb{Z}} \beta_{n}^{i_{n-r m}}
$$


holds for every $m \in \mathbb{Z}$. This holds trivially for $m=0$. Assume now that $m>0$ and (5.2) holds for $m-1$. Since Fin $\left(\mathscr{L}_{k}\right)$ is abelian, we get

$$
\begin{aligned}
\beta_{m} \varphi_{\left(i_{n}\right)_{n},\left(j_{n}\right)_{n}, r} & =\left(\prod_{n \in \mathbb{Z}} \beta_{n}^{j_{n}}\right) \xi^{r}\left(\prod_{n \in \mathbb{Z}} \beta_{n}^{i_{n-r(m-1)}}\right) \xi^{-r}\left(\prod_{n \in \mathbb{Z}} \beta_{n}^{-j_{n}}\right) \\
& =\xi^{r}\left(\prod_{n \in \mathbb{Z}} \beta_{n}^{i_{n-r(m-1)}}\right) \xi^{-r} \\
& =\prod_{n \in \mathbb{Z}} \beta_{n+r}^{i_{n-r(m-1)}}=\prod_{n \in \mathbb{Z}} \beta_{n}^{i_{n-r m}} .
\end{aligned}
$$

Thus (5.2) holds for every $m>0$. Similarly, we show that it holds for $m<0$.

Now we can derive

$$
\beta_{m} \varphi_{\left(i_{n}\right)_{n},\left(j_{n}\right)_{n}, r} \varphi_{\left(i_{n}^{\prime}\right)_{n},\left(j_{n}^{\prime}\right)_{n}, r^{\prime}}=\prod_{n \in \mathbb{Z}} \beta_{n}^{\sum_{k \in \mathbb{Z}} i_{n-r^{\prime} k}^{\prime} i_{k-r m}} .
$$

Indeed, using (5.2) twice, we get

$$
\begin{aligned}
\beta_{m} \varphi_{\left(i_{n}\right)_{n},\left(j_{n}\right)_{n}, r} \varphi_{\left(i_{n}^{\prime}\right)_{n},\left(j_{n}^{\prime}\right)_{n}, r^{\prime}} & =\prod_{k \in \mathbb{Z}} \beta_{k}^{i_{k-r m}} \varphi_{\left(i_{n}^{\prime}\right)_{n},\left(j_{n}^{\prime}\right)_{n}, r^{\prime}} \\
& =\prod_{k \in \mathbb{Z}}\left(\prod_{n \in \mathbb{Z}} \beta_{n}^{i_{n-r^{\prime} k}^{\prime}}\right)^{i_{k-r m}} \\
& =\prod_{n \in \mathbb{Z}} \beta_{n}^{\sum_{k \in \mathbb{Z}} i_{n-r^{\prime} k}^{\prime} i_{k-r m}} .
\end{aligned}
$$

It follows easily from (5.3) that

$$
\varphi_{\left(i_{n}\right)_{n},(0)_{n}, 1} \varphi_{\left(i_{n}^{\prime}\right)_{n},(0)_{n}, 1}=\varphi_{\left(i_{n}^{\prime}\right)_{n},(0)_{n}, 1} \varphi_{\left(i_{n}\right)_{n},(0)_{n}, 1} .
$$

Indeed, $\xi$ is fixed by both endomorphisms, and

$$
\begin{aligned}
\alpha \varphi_{\left(i_{n}\right)_{n},(0)_{n}, 1} \varphi_{\left(i_{n}^{\prime}\right)_{n},(0)_{n}, 1} & =\prod_{n \in \mathbb{Z}} \beta_{n}^{\sum_{k \in \mathbb{Z}} i_{n-k}^{\prime} i_{k}}=\prod_{n \in \mathbb{Z}} \beta_{n}^{\sum_{k \in \mathbb{Z}} i_{k}^{\prime} i_{n-k}} \\
& =\alpha \varphi_{\left(i_{n}^{\prime}\right)_{n},(0)_{n}, 1} \varphi_{\left(i_{n}\right)_{n},(0)_{n}, 1} .
\end{aligned}
$$

We also get

$$
\varphi_{\left(i_{n}\right)_{n},(0)_{n}, 1} \varphi_{\left(i_{n}^{\prime}\right)_{n},(0)_{n}, 1}=\varphi_{\left(\sum_{k \in \mathbb{Z}} i_{n-k}^{\prime} i_{k}\right)_{n},(0)_{n}, 1} .
$$

This formula can be generalized for an arbitrary number of factors. For every $q \geq 2$, we have

$$
\varphi_{\left(i_{n}^{(1)}\right)_{n},(0)_{n}, 1} \ldots \varphi_{\left(i_{n}^{(q)}\right)_{n},(0)_{n}, 1}=\varphi_{\left(\sum_{k_{1}+\cdots+k_{q}=n} i_{k_{1}}^{(1)} \ldots i_{k_{q}}^{(q)}\right)_{n},(0)_{n}, 1},
$$


where the $k_{j}$ take values in $\mathbb{Z}$. Indeed, the case $q=2$ is just (5.5) rewritten, so we assume that (5.6) holds for $q \geq 2$, and we prove it for $q+1$. Using (5.5) and the induction hypothesis, we get

$$
\begin{aligned}
& p_{\left(i_{n}^{(1)}\right)_{n},(0)_{n}, 1} \ldots \varphi_{\left(i_{n}^{(q+1)}\right)_{n},(0)_{n}, 1} \\
& \quad=\varphi_{\left(\sum_{k_{1}+\cdots+k_{q}=n} i_{k_{1}}^{(1)} \ldots i_{k_{q}}^{(q)}\right)_{n},(0)_{n}, 1} \varphi_{\left(i_{n}^{(q+1)}\right)_{n},(0)_{n}, 1} \\
& \quad=\varphi_{\left(\sum_{k_{q+1} \in \mathbb{Z}}\left(\sum_{k_{1}+\cdots+k_{q}=n-k_{q+1}} i_{k_{1}}^{(1)} \ldots i_{k_{q}}^{(q)}\right) i_{k_{q+1}}^{(q+1)}\right)_{n},(0)_{n}, 1} \\
& \quad=\varphi_{\left(\sum_{k_{1}+\cdots+k_{q+1}=n} i_{k_{1}}^{(1)} \ldots i_{k_{q+1}}^{(q+1)}\right)_{n},(0)_{n}, 1},
\end{aligned}
$$

and so (5.6) holds.

\subsection{Stabilizers in the lamplighter group and their generating sets}

It is much harder to identify the automorphisms of $\mathscr{L}_{k}$. We start from studying the following subgroup of $\operatorname{Aut}\left(\mathscr{L}_{k}\right)$ :

$$
\operatorname{Stab}_{\mathscr{L}_{k}}(\xi)=\left\{\varphi \in \operatorname{Aut}\left(\mathscr{L}_{k}\right) \mid \xi \varphi=\xi\right\} .
$$

It is straightforward to check that (5.5) is equivalent to the following, for given $\varphi, \varphi^{\prime} \in \operatorname{Stab}_{\mathscr{L}_{k}}(\xi)$ :

$$
\begin{gathered}
\text { if } \alpha \varphi=\prod_{j=1}^{r} \beta_{m_{j}}^{i_{j}} \quad \text { and } \alpha \varphi^{\prime}=\prod_{\ell=1}^{s} \beta_{n_{\ell}}^{i_{\ell}^{\prime}}, \\
\text { then } \alpha \varphi \varphi^{\prime}=\prod_{j=1}^{r} \prod_{\ell=1}^{s} \beta_{m_{j}+n_{\ell}}^{i_{j} i_{\ell}^{\prime}} .
\end{gathered}
$$

We introduce some notation for specific automorphisms in $\operatorname{Stab}_{\mathscr{L}_{k}}(\xi)$. Let $\left(\varepsilon_{n}\right)_{n}$ be the function from $\mathbb{Z}$ to $\mathbb{Z}_{k}$ defined by

$$
\varepsilon_{n}= \begin{cases}1 & \text { if } n=0, \\ 0 & \text { otherwise }\end{cases}
$$

This function will be handy throughout the rest of the section. Let $\lambda$ denote the inner automorphism of $\mathscr{L}_{k}$ defined by $x \lambda=\xi x \xi^{-1}$. Clearly, $\lambda \in \operatorname{Stab}_{\mathscr{L}_{k}}(\xi)$.

For every $j \in \mathbb{Z}_{k}^{*}$, we define $\eta_{j} \in \operatorname{Stab}_{\mathscr{L}_{k}}(\xi)$ by $\alpha \eta_{j}=\alpha^{j}$. Note that $\alpha^{j}$ is well defined since $\alpha^{k}=1$. If $j^{-1}$ denotes the inverse of $j$ in $\mathbb{Z}_{k}^{*}$, then we have $\eta_{j} \eta_{j^{-1}}=1=\eta_{j^{-1}} \eta_{j}$, and so $\eta_{j}$ is indeed an automorphism. 
Finally, let $m \in \mathbb{Z}$ and $j \in \mathbb{Z}_{k}^{*}$. We define $\gamma_{m, j} \in \operatorname{Stab}_{\mathscr{L}_{k}}(\xi)$ by $\alpha \gamma_{m, j}=\beta_{m}^{j}$. Note that

$$
\lambda=\gamma_{1,1} \quad \text { and } \quad \eta_{j}=\gamma_{0, j} .
$$

Since $\alpha \lambda^{m}=\beta_{m}$ holds for every $m \in \mathbb{Z}$, it follows easily that

$$
\gamma_{m, j}=\lambda^{m} \eta_{j}
$$

Lemma 5.1. Let $p$ be a positive prime. Then

$$
\operatorname{Stab}_{\mathscr{L}_{p}}(\xi)=\left\{\gamma_{m, j} \mid m \in \mathbb{Z}, j \in \mathbb{Z}_{p}^{*}\right\}=\left\langle\lambda, \eta_{2}, \ldots, \eta_{p-1}\right\rangle .
$$

Proof. We had already established that $\gamma_{m, j} \in \operatorname{Stab}_{\mathscr{L}_{p}}(\xi)$ for all $m, j$. Conversely, let $\psi \in \operatorname{Stab}_{\mathscr{L}_{p}}(\xi)$. Then we may write $\psi=\varphi_{\left(i_{n}\right)_{n},(0)_{n}, 1}$ for some function $\left(i_{n}\right)_{n}$ from $\mathbb{Z}$ to $\mathbb{Z}_{k}$ with finite support. As $\xi \psi^{-1}=\xi$, we may write $\psi^{-1}=\varphi_{\left(i_{n}^{\prime}\right)_{n},(0)_{n}, 1}$ for some $\left(i_{n}^{\prime}\right)_{n}$. Since $\alpha \psi \psi^{-1}=\alpha$, it follows from (5.3) that

$$
\sum_{k \in \mathbb{Z}} i_{n-k}^{\prime} i_{k}= \begin{cases}1 & \text { if } n=0 \\ 0 & \text { otherwise }\end{cases}
$$

Assume that

$$
\operatorname{supp}\left(\left(i_{n}\right)_{n}\right)=\left\{n \in \mathbb{Z} \mid i_{n} \neq 0\right\}=\left\{s_{1}, \ldots, s_{m}\right\} \quad \text { with } s_{1}<\cdots<s_{m}
$$

Assume also that $\operatorname{supp}\left(\left(i_{n}^{\prime}\right)_{n}\right)=\left\{s_{1}^{\prime}, \ldots, s_{m^{\prime}}^{\prime}\right\}$ with $s_{1}^{\prime}<\cdots<s_{m^{\prime}}^{\prime}$. Then $k>s_{1}$ if and only if $s_{1}+s_{1}^{\prime}-k<s_{1}^{\prime}$; hence

$$
\sum_{k \in \mathbb{Z}} i_{s_{1}+s_{1}^{\prime}-k}^{\prime} i_{k}=i_{s_{1}^{\prime}}^{\prime} i_{s_{1}} \neq 0 .
$$

Similarly, $k<s_{m}$ if and only if $s_{m}+s_{m^{\prime}}^{\prime}-k>s_{m^{\prime}}^{\prime}$; hence

$$
\sum_{k \in \mathbb{Z}} i_{s_{m}+s_{m^{\prime}}^{\prime}-k}^{\prime} i_{k}=i_{s_{m^{\prime}}^{\prime}}^{\prime} i_{s_{m}} \neq 0 .
$$

In view of (5.10), we get $s_{1}+s_{1}^{\prime}=s_{m}+s_{m^{\prime}}^{\prime}$; hence $m=m^{\prime}=1$, so $\alpha \psi=\beta_{s_{1}}$. Thus $\psi=\gamma_{s_{1}, i_{s_{1}}}$, and the first equality of the lemma is established. The second follows from (5.8) and (5.9).

We consider now the case of powers of primes. Let $p$ be a positive prime and $s \geq 2$. Given $m \in \mathbb{Z} \backslash\{0\}$ and $r \in \mathbb{Z}_{p^{s}}$, we define an endomorphism $\delta_{m, p r}$ of $\mathscr{L}_{p^{s}}$ by $\alpha \delta_{m, p r}=\alpha \beta_{m}^{p r}$. 
Lemma 5.2. Let $p$ be a positive prime and $s \geq 2$. Then

$$
\begin{aligned}
\operatorname{Stab}_{\mathscr{L}_{p^{s}}}(\xi) & =\left\{\varphi_{\left(i_{n}\right)_{n},(0)_{n}, 1} \mid \text { there exists a unique } m \in \mathbb{Z} \text { such that } p \nmid i_{m}\right\} \\
& =\left\langle\lambda, \eta_{j}, \delta_{m, p r} \mid j \in \mathbb{Z}_{p^{s}}^{*}, m \in \mathbb{Z} \backslash\{0\}, r \in \mathbb{Z}_{p^{s}}\right\rangle .
\end{aligned}
$$

Proof. We begin the proof by proving some inclusions as separate claims to clarify the proof path.

\section{Claim 1. We have}

$$
\operatorname{Stab}_{\mathscr{L}_{p} s}(\xi) \subseteq\left\{\varphi_{\left(i_{n}\right)_{n},(0)_{n}, 1} \mid \text { there exists a unique } m \in \mathbb{Z} \text { such that } p \nmid i_{m}\right\} .
$$

Proof of Claim 1. Let $\psi=\varphi_{\left(i_{n}\right)_{n},(0)_{n}, 1} \in \operatorname{Stab}_{\mathscr{L}_{p} s}(\xi)$. Write $\psi^{-1}=\varphi_{\left(i_{n}^{\prime}\right)_{n},(0)_{n}, 1}$. In view of (5.3), (5.10) holds; therefore we have $\sum_{k \in \mathbb{Z}} i_{-k}^{\prime} i_{k}=1\left(\bmod p^{s}\right)$, and thus $\sum_{k \in \mathbb{Z}} i_{-k}^{\prime} i_{k}=1(\bmod p)$. It follows that there exists some $m \in \mathbb{Z}$ such that $p$ does not divide $i_{-m}^{\prime} i_{m}$. We may assume that $r$ and $m$ are respectively the leftmost and the rightmost integers $n \in \mathbb{Z}$ such that $p$ does not divide $i_{n}$, so that $r \leq m$. Let $r^{\prime}$ and $m^{\prime}$ be respectively the leftmost and the rightmost integers $n \in \mathbb{Z}$ such that $p$ does not divide $i_{n}^{\prime}$, so that $r^{\prime} \leq m^{\prime}$.

We claim that $\sum_{k \in \mathbb{Z}} i_{m^{\prime}+m-k}^{\prime} i_{k} \neq 0(\bmod p)$. Indeed, if $k>m$, then $p$ divides $i_{k}$, and $p$ divides $i_{m^{\prime}+m-k}^{\prime}$ if $k<m$ (equivalent to $m^{\prime}+m-k>m^{\prime}$ ). However, $p$ does not divide $i_{m^{\prime}+m-m}^{\prime} i_{m}$ since both factors are invertible in $\mathbb{Z}_{p^{s}}$. Thus $\sum_{k \in \mathbb{Z}} i_{m^{\prime}+m-k}^{\prime} i_{k} \neq 0(\bmod p)$. Similarly, $\sum_{k \in \mathbb{Z}} i_{r^{\prime}+r-k}^{\prime} i_{k} \neq 0(\bmod p)$. In view of (5.10), we get $m^{\prime}+m=r^{\prime}+r$. Hence $m^{\prime}+m=r^{\prime}+r \leq m^{\prime}+r$, yielding $m \leq r$ and consequently $m=r$. Therefore, there exists a unique $m \in \mathbb{Z}$ such that $p$ does not divide $i_{m}$.

Claim 2. $\left\langle\lambda, \eta_{j}, \delta_{m, p r} \mid j \in \mathbb{Z}_{k}^{*}, m \in \mathbb{Z} \backslash\{0\}, r \in \mathbb{Z}_{p^{s}}\right\rangle \subseteq \operatorname{Stab}_{\mathscr{L}_{p^{s}}}(\xi)$.

Proof of Claim 2. Let us now fix an $m \in \mathbb{Z} \backslash\{0\}$ and an $r \in \mathbb{Z}_{p^{s}}$ to show that $\delta_{m, p r} \in \operatorname{Stab}_{\mathscr{L}_{p} s}(\xi)$. In order to do so, we introduce

$$
\Phi_{m}=\left\{\varphi_{\left(i_{n}\right)_{n},(0)_{n}, 1} \mid i_{0}=1 \text { and } i_{n}=0 \text { if } \frac{n}{m} \notin \mathbb{N}\right\} .
$$

We claim that $\Phi_{m}$ is a submonoid of endomorphisms of $\mathscr{L}_{p^{s}}$. It certainly contains the identity. Let $i_{n}^{\prime \prime}=\sum_{k \in \mathbb{Z}} i_{n-k}^{\prime} i_{k}$. By (5.5), we have

$$
\varphi_{\left(i_{n}\right)_{n},(0)_{n}, 1} \varphi_{\left(i_{n}^{\prime}\right)_{n},(0)_{n}, 1}=\varphi_{\left(i_{n}^{\prime \prime}\right)_{n},(0)_{n}, 1} .
$$

Now $i_{0}^{\prime \prime}=\sum_{k \in \mathbb{Z}} i_{-k}^{\prime} i_{k}$. If $k \neq 0$, then $\frac{-k}{m} \notin \mathbb{N}$ or $\frac{k}{m} \notin \mathbb{N}$; hence $i_{-k}^{\prime} i_{k}=0$. It follows that $i_{0}^{\prime \prime}=i_{0}^{\prime} i_{0}=1$. Suppose now that $\frac{n}{m} \notin \mathbb{N}$. For every $k \in \mathbb{Z}$, we have $\frac{n-k}{m} \notin \mathbb{N}$ or $\frac{k}{m} \notin \mathbb{N}$; hence $i_{n-k}^{\prime} i_{k}=0$, and so $i_{n}^{\prime \prime}=0$. Thus $\Phi_{m}$ is a monoid. 
We define now a function $\Lambda$ from $\Phi_{m}$ to the polynomial ring $\mathbb{Z}_{p^{s}}[x]$ as follows. Given $\varphi_{\left(i_{n}\right)_{n},(0)_{n}, 1} \in \Phi_{m}$, let

$$
\varphi_{\left(i_{n}\right)_{n},(0)_{n}, 1} \Lambda=\sum_{k \in \mathbb{N}} i_{m k} x^{k} .
$$

Since $i_{n}$ can only be nonzero for $n \in m \mathbb{N}$, the function $\Lambda$ is injective. We show that it is a monoid endomorphism with respect to the multiplicative structure of $\mathbb{Z}_{p^{s}}[x]$. Clearly, it preserves the identity. Consider now an equality

$$
\varphi_{\left(i_{n}\right)_{n},(0)_{n}, 1} \varphi_{\left(i_{n}^{\prime}\right)_{n},(0)_{n}, 1}=\varphi_{\left(i_{n}^{\prime \prime}\right)_{n},(0)_{n}, 1}
$$

in $\Phi_{m}$. We must show that

$$
\left(\sum_{k \in \mathbb{N}} i_{m k} x^{k}\right)\left(\sum_{k \in \mathbb{N}} i_{m k}^{\prime} x^{k}\right)=\sum_{k \in \mathbb{N}} i_{m k}^{\prime \prime} x^{k},
$$

which is equivalent to

$$
\sum_{k_{1}, k_{2} \in \mathbb{N}} i_{m k_{1}} i_{m k_{2}}^{\prime} x^{k_{1}+k_{2}}=\sum_{k \in \mathbb{N}} i_{m k}^{\prime \prime} x^{k}
$$

and therefore to

$$
\sum_{j=0}^{k} i_{m j} i_{m k-m j}^{\prime}=i_{m k}^{\prime \prime}
$$

holding for all $k \in \mathbb{N}$.

On the other hand, it follows from (5.5) and (5.11) that

$$
i_{m k}^{\prime \prime}=\sum_{\ell \in \mathbb{Z}} i_{m k-\ell}^{\prime} i_{\ell}
$$

Since $i_{n}=i_{n}^{\prime}=0$ if $\frac{n}{m} \notin \mathbb{N},(5.13)$ implies (5.12), and so $\Lambda$ is a monoid homomorphism.

We prove next that

$$
\delta_{m, p r}^{p^{s}}=1 .
$$

Since $\Lambda$ is an injective monoid homomorphism, it suffices to show that

$$
\left(\delta_{m, p r} \Lambda\right)^{p^{s}}=1, \quad \text { i.e. }(1+p r x)^{p^{s}}=1 .
$$

In view of Newton's binomial theorem, this is equivalent to

$$
\sum_{j=1}^{p^{s}}\left(\begin{array}{c}
p^{s} \\
j
\end{array}\right)(p r x)^{j}=0 .
$$


Thus it suffices to show that

$$
p^{s} \mid\left(\begin{array}{c}
p^{s} \\
j
\end{array}\right) p^{j}
$$

for $j=1, \ldots, p^{s}$.

Given $n \in \mathbb{Z} \backslash\{0\}$, let

$$
n v_{p}=\max \left\{k \in \mathbb{N} \mid p^{k} \text { divides } n\right\} .
$$

Write also $0 v_{p}=\infty$. Given $j \in\left\{1, \ldots, p^{s}\right\}$, we can write

$$
\left(\begin{array}{c}
p^{s} \\
j
\end{array}\right) p^{j}=p^{s} \frac{p^{s}-1}{1} \frac{p^{s}-2}{2} \ldots \frac{p^{s}-(j-1)}{j-1} \frac{p^{j}}{j} .
$$

Since $\left(p^{s}-i\right) v_{p}=i v_{p}$ for $i=1, \ldots, j-1$, it follows that $p$ does not divide neither the numerator nor the denominator of $\frac{p^{s}-i}{i_{p^{j}}}$, and since $j v_{p}<j, p$ divides the numerator but not the denominator of $\frac{p^{j}}{j}$. Since both sides of equation (5.16) represent a positive integer, (5.15) holds and so does (5.14). Therefore, $\delta_{m, p r} \in \operatorname{Stab}_{\mathscr{L}_{p} s}(\xi)$.

So far, Claims 1 and 2 established that

$\operatorname{Stab}_{\mathscr{L}_{p} s}(\xi) \subseteq\left\{\varphi_{\left(i_{n}\right)_{n},(0)_{n}, 1} \mid\right.$ there exists a unique $m \in \mathbb{Z}$ such that $\left.p \nmid i_{m}\right\}$ and

$$
\left\langle\lambda, \eta_{j}, \delta_{m, p r} \mid j \in \mathbb{Z}_{k}^{*}, m \in \mathbb{Z} \backslash\{0\}, r \in \mathbb{Z}_{p^{s}}\right\rangle \subseteq \operatorname{Stab}_{\mathscr{L}_{p^{s}}}(\xi) .
$$

To complete the proof of the lemma, we show the following claim.

Claim 3. For every $\varphi_{\left(i_{n}\right)_{n},(0)_{n}, 1}$ such that there exists a unique $m \in \mathbb{Z}$ with $p$ not dividing $i_{m}$, there exists some $\theta \in\left\langle\lambda, \eta_{j}, \delta_{m, p r} \mid j \in \mathbb{Z}_{k}^{*}, m \in \mathbb{Z} \backslash\{0\}, r \in \mathbb{Z}_{p^{s}}\right\rangle$ such that $\varphi_{\left(i_{n}\right)_{n},(0)_{n}, 1} \theta=1$.

Proof of Claim 3. Up to replacing $\varphi_{\left(i_{n}\right)_{n},(0)_{n}, 1}$ by $\varphi_{\left(i_{n}\right)_{n},(0)_{n}, 1} \lambda^{-m} \eta_{i_{m}^{-1}}$ if needed, we may assume that $i_{0}=1$ from now on.

We use a double induction scheme. Let $\psi=\varphi_{\left(i_{n}\right)_{n},(0)_{n}, 1}$, and define

$$
t_{\psi}=\min \left\{i_{n} v_{p} \mid n \in \mathbb{Z} \backslash\{0\}\right\}, \quad u_{\psi}=\left|\left\{n \in \mathbb{Z} \backslash\{0\} \mid i_{n} v_{p}=t_{\psi}\right\}\right| .
$$

We start by considering the case $t_{\psi} \geq \frac{s}{2}$. Take $r \in \mathbb{Z}$ such that $i_{r} v_{p}=t_{\psi}$, and let $\psi^{\prime}=\psi \delta_{r,-i_{r}}$. By (5.5), we have

$$
\psi^{\prime}=\varphi_{\left(i_{n}-i_{r} i_{n-r}\right)_{n},(0)_{n}, 1}
$$


Let $s^{\prime}=\left\lceil\frac{s}{2}\right\rceil$. If $n-r \neq 0$, then $p^{s^{\prime}}$ divides both $i_{r}$ and $i_{n-r}$; hence $i_{r} i_{n-r}=0$. But if $n=r$, then $i_{n}-i_{r} i_{n-r}=0$; hence $\psi^{\prime}$ is obtained from $\psi$ by replacing $i_{r}$ by 0 . Applying successively this procedure, we end up by obtaining the identity function; hence the claim holds in the case $t_{\psi} \geq \frac{s}{2}$.

We consider now the case $t_{\psi}=t<\frac{s}{2}$, and we assume that the claim holds for all $\psi^{\prime}$ such that $t_{\psi^{\prime}}>t$. Take $r \in \mathbb{Z}$ such that $i_{r} v_{p}=t_{\psi}$, and let $\psi^{\prime}=\psi \delta_{r,-i_{r}}$. By (5.5), we have

$$
\psi^{\prime}=\varphi\left(i_{n}-i_{r} i_{n-r}\right)_{n},(0)_{n}, 1 \cdot
$$

If $n=r$, then $i_{n}-i_{r} i_{n-r}=0$. If $n \neq r$, then

$$
\begin{aligned}
\left(i_{n}-i_{r} i_{n-r}\right) v_{p} & \geq \min \left\{\left(i_{n} v_{p},\left(i_{r} i_{n-r}\right) v_{p}\right)\right\}=\min \left\{\left(i_{n} v_{p}, i_{r} v_{p}+i_{n-r} v_{p}\right)\right\} \\
& \geq \min \left\{\left(i_{n} v_{p}, 2 t_{\psi}\right)\right\}
\end{aligned}
$$

hence either $\psi^{\prime}=1$, or $t_{\psi^{\prime}}>t_{\psi}$, or $t_{\psi^{\prime}}=t_{\psi}$ and $u_{\psi^{\prime}}<u_{\psi}$. Therefore, the claim follows by induction.

By putting together Claims 1, 2 and 3, we finish the proof of Lemma 5.2.

Lemma 5.3. Let $p$ be a positive prime and $s \geq 2$. Then $\operatorname{Stab}_{\mathscr{L}_{p} s}(\xi)$ is not finitely generated.

Proof. Suppose that $\operatorname{Stab}_{\mathscr{L}_{p^{s}}}(\xi)$ is finitely generated. In view of Lemma 5.2, it admits a generating set of the form $A_{M}$, where

$$
A_{M}=\left\{\lambda, \eta_{j}, \delta_{m, p r} \mid j \in \mathbb{Z}_{k}^{*},-M \leq m \leq M, r \in \mathbb{Z}_{p^{s}}\right\}
$$

for some $M \geq 1$.

Since $\operatorname{Stab}_{\mathscr{L}_{p} s}(\xi)$ is abelian by (5.4), we may write $\delta_{M s, p}=\psi \lambda^{t}$ for some $\psi \in\left\langle A_{M} \backslash\{\lambda\}\right\rangle$ and $t \in \mathbb{Z}$. Thus

$$
\alpha \psi=\alpha \delta_{M s, p} \lambda^{-t}=\xi^{-t}\left(\alpha \beta_{M s}^{p}\right) \xi^{t}=\beta_{-t} \beta_{M s-t}^{p} .
$$

Since the set of automorphisms $\varphi_{\left(i_{n}\right)_{n},(0)_{n}, 1}$ satisfying $i_{0} \in \mathbb{Z}_{p^{s}}^{*}$ contains $A_{M}$ and is closed under composition in view of (5.5), it follows that $t=0$, and so

$$
\delta_{M s, p} \in\left\langle A_{M} \backslash\{\lambda\}\right\rangle .
$$

Using (5.4) once again, we may write

$$
\delta_{M s, p}=\varphi_{\left(i_{n}^{(1)}\right)_{n},(0)_{n}, 1} \cdots \varphi_{\left(i_{n}^{(q)}\right)_{n},(0)_{n}, 1}
$$


for some $\varphi\left(i_{n}^{(1)}\right)_{n},(0)_{n}, 1, \ldots, \varphi_{\left(i_{n}^{(q)}\right)_{n},(0)_{n}, 1}$ (note that $A_{M} \backslash\{\lambda\}$ is closed under inversion in view of (5.14)). By (5.6), we get

$$
\delta_{M s, p}=\varphi_{\left(\sum_{k_{1}+\cdots+k_{q}=n} i_{k_{1}}^{(1)} \ldots i_{k q}^{(q)}\right)_{n},(0)_{n}, 1} ;
$$

hence comparing the $M s$ components yields

$$
p=\sum_{k_{1}+\cdots+k_{q}=M s} i_{k_{1}}^{(1)} \ldots i_{k_{q}}^{(q)} .
$$

It follows that $i_{k_{1}}^{(1)} \ldots i_{k_{q}}^{(q)} \neq 0$ for some $k_{1}, \ldots, k_{q} \in \mathbb{Z}$ satisfying

$$
k_{1}+\cdots+k_{q}=M s .
$$

In particular, $i_{k_{1}}^{(1)}, \ldots, i_{k_{q}}^{(q)} \neq 0$. Given the structure of the elements of $A_{M} \backslash\{\lambda\}$, it follows that $-M \leq k_{1}, \ldots, k_{q} \leq M$. Since $k_{1}+\cdots+k_{q}=M s$, there exist at least $s$ nonzero elements among $k_{1}, \ldots, k_{q}$, and so it follows that $p$ divides at least $s$ elements among $i_{k_{1}}^{(1)}, \ldots, i_{k_{q}}^{(q)}$, and so $i_{k_{1}}^{(1)} \ldots i_{k_{q}}^{(q)}=0$, a contradiction. Therefore, $\operatorname{Stab}_{\mathscr{L}_{p} s}(\xi)$ is not finitely generated.

\subsection{Automorphisms of the lamplighter group}

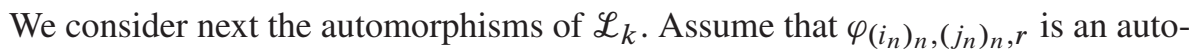
morphism of $\mathscr{L}_{k}$. Since $\operatorname{Fin}\left(L_{k}\right) \cup\left\{\xi^{r}\right\}$ generates a proper subgroup of $\mathscr{L}_{k}$ unless $r= \pm 1$, we can write

$$
\operatorname{Aut}\left(\mathscr{L}_{k}\right)=\operatorname{Aut}_{+}\left(\mathscr{L}_{k}\right) \cup \operatorname{Aut}_{-}\left(\mathscr{L}_{k}\right),
$$

where Aut $+\left(\mathscr{L}_{k}\right)$ denotes the set of automorphisms of the form $\varphi_{\left(i_{n}\right)_{n},\left(j_{n}\right)_{n}, 1}$ (the positive automorphisms) and Aut_ $\left(\mathscr{L}_{k}\right)$ denotes the set of automorphisms of the form $\varphi_{\left(i_{n}\right)_{n},\left(j_{n}\right)_{n},-1}$ (the negative automorphisms). Let $\zeta$ be the endomorphism of $\mathscr{L}_{k}$ defined by $\alpha \zeta=\alpha$ and $\xi \zeta=\xi^{-1}$. It follows that $\zeta \in$ Aut $_{-}\left(\mathscr{L}_{k}\right)$. It is easy to check that $\operatorname{Aut}_{+}\left(\mathscr{L}_{k}\right)$ is a (normal) subgroup of index 2 of $\operatorname{Aut}\left(\mathscr{L}_{k}\right)$ containing $\operatorname{Stab}_{\mathscr{L}_{k}}(\xi)$. Thus

$$
\operatorname{Aut}\left(\mathscr{L}_{k}\right) \cong \operatorname{Aut}_{+}\left(\mathscr{L}_{k}\right) \rtimes C_{2}
$$

Lemma 5.4. Let $k \geq 2$. Then

$$
\sigma: \operatorname{Aut}_{+}\left(\mathscr{L}_{k}\right) \rightarrow \operatorname{Stab}_{\mathscr{L}_{k}}(\xi), \quad \varphi_{\left(i_{n}\right)_{n},\left(j_{n}\right)_{n}, 1} \mapsto \varphi_{\left(i_{n}\right)_{n},(0)_{n}, 1}
$$

is a surjective group homomorphism. 
Proof. Let $\varphi_{\left(i_{n}\right)_{n},\left(j_{n}\right)_{n}, 1}, \varphi_{\left(i_{n}^{\prime}\right)_{n},\left(j_{n}^{\prime}\right)_{n}, 1} \in$ Aut $_{+}\left(\mathscr{L}_{k}\right)$. By (5.3), we have

$$
\begin{aligned}
\left(\varphi_{\left(i_{n}\right)_{n},\left(j_{n}\right)_{n}, 1} \varphi_{\left(i_{n}^{\prime}\right)_{n},\left(j_{n}^{\prime}\right)_{n}, 1}\right) \sigma & =\varphi_{\left(\sum_{q \in \mathbb{Z}} i_{n-q}^{\prime} i_{q}\right)_{n},(0)_{n}, 1}=\varphi_{\left(i_{n}\right)_{n},(0)_{n}, 1} \varphi_{\left(i_{n}^{\prime}\right)_{n},(0)_{n}, 1} \\
& =\left(\varphi_{\left(i_{n}\right)_{n},\left(j_{n}\right)_{n}, 1} \sigma\right)\left(\varphi_{\left(i_{n}^{\prime}\right)_{n},\left(j_{n}^{\prime}\right)_{n}, 1} \sigma\right) .
\end{aligned}
$$

Hence $\sigma$ is a monoid endomorphism from $\operatorname{Aut}_{+}\left(\mathscr{L}_{k}\right)$ into the monoid of endomorphisms of $\mathscr{L}_{k}$ fixing $\xi$. If we take $\varphi_{\left(i_{n}^{\prime}\right)_{n},\left(j_{n}^{\prime}\right)_{n}, 1}=\varphi_{\left(i_{n}\right)_{n},\left(j_{n}\right)_{n}, 1}^{-1}$, it follows that

$$
\left(\varphi_{\left(i_{n}\right)_{n},\left(j_{n}\right)_{n}, 1} \sigma\right)\left(\varphi_{\left(i_{n}^{\prime}\right)_{n},\left(j_{n}^{\prime}\right)_{n}, 1} \sigma\right)=1
$$

Similarly,

$$
\left(\varphi_{\left(i_{n}^{\prime}\right)_{n},\left(j_{n}^{\prime}\right)_{n}, 1} \sigma\right)\left(\varphi_{\left(i_{n}\right)_{n},\left(j_{n}\right)_{n}, 1} \sigma\right)=1
$$

Therefore, $\sigma$ is a (group) homomorphism from $\operatorname{Aut}_{+}\left(\mathscr{L}_{k}\right)$ into $\operatorname{Stab}_{\mathscr{L}_{k}}(\xi)$. Since $\sigma$ fixes all the automorphisms from $\operatorname{Stab}_{\mathscr{L}_{k}}(\xi)$, it is surjective.

Lemma 5.5. Let $k \geq 2$ and $r \in \mathbb{Z}$. Let $\left(i_{n}\right)_{n}$ and $\left(j_{n}\right)_{n}$ be functions with finite support from $\mathbb{Z}$ to $\mathbb{Z}_{k}$. Then the following conditions are equivalent:

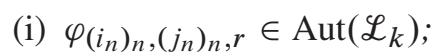

(ii) $\varphi_{\left(i_{n}\right)_{n},(0)_{n}, 1} \in \operatorname{Stab}_{\mathscr{L}_{k}}(\xi)$ and $r= \pm 1$.

Proof. (i) $\Rightarrow$ (ii). We have already remarked that (i) implies $r= \pm 1$. If $r=1$, then $\varphi_{\left(i_{n}\right)_{n},(0)_{n}, 1} \in \operatorname{Stab}_{\mathscr{L}_{k}}(\xi)$ follows from Lemma 5.4. Hence we may assume that $r=-1$. Composing with $\zeta=\varphi_{\left(\varepsilon_{n}\right)_{n},(0)_{n},-1}$, we get

$$
\begin{aligned}
& \alpha \varphi_{\left(i_{n}\right)_{n},\left(j_{n}\right)_{n},-1} \zeta=\left(\prod_{n \in \mathbb{Z}} \beta_{n}^{i_{n}}\right) \zeta=\prod_{n \in \mathbb{Z}} \beta_{-n}^{i_{n}}=\prod_{n \in \mathbb{Z}} \beta_{n}^{i_{-n}}, \\
& \xi \varphi_{\left(i_{n}\right)_{n},\left(j_{n}\right)_{n},-1} \zeta=\left(\left(\prod_{n \in \mathbb{Z}} \beta_{n}^{j_{n}}\right) \xi^{-1}\right) \zeta=\left(\prod_{n \in \mathbb{Z}} \beta_{-n}^{j_{n}}\right) \xi=\left(\prod_{n \in \mathbb{Z}} \beta_{n}^{j_{-n}}\right) \xi
\end{aligned}
$$

hence $\varphi_{\left(i_{n}\right)_{n},\left(j_{n}\right)_{n},-1} \zeta=\varphi_{\left(i_{-n}\right)_{n},\left(j_{-n}\right)_{n}, 1} \in \operatorname{Aut}_{+}\left(\mathscr{L}_{k}\right)$, and so

$$
\varphi_{\left(i_{-n}\right)_{n},(0)_{n}, 1} \in \operatorname{Stab}_{\mathscr{L}_{k}}(\xi)
$$

by the preceding case. Let $\varphi_{\left(i_{n}^{\prime}\right)_{n},(0)_{n}, 1}=\varphi_{\left(i_{-n}\right)_{n},(0)_{n}, 1}^{-1}$. It follows from (5.3) that

$$
\sum_{k \in \mathbb{Z}} i_{n-k}^{\prime} i_{-k}= \begin{cases}1 & \text { if } n=0 \\ 0 & \text { otherwise }\end{cases}
$$

But then, for $i_{n}^{\prime \prime}=i_{-n}^{\prime}$, we get

$$
\sum_{k \in \mathbb{Z}} i_{-n-k}^{\prime \prime} i_{k}= \begin{cases}1 & \text { if } n=0 \\ 0 & \text { otherwise }\end{cases}
$$

Replacing $-n-k$ by $n-k$, we get $\varphi_{\left(i_{n}^{\prime \prime}\right)_{n},(0)_{n}, 1}=\varphi_{\left(i_{n}\right)_{n},(0)_{n}, 1}^{-1}$, and so (ii) holds. 
(ii) $\Rightarrow$ (i). Assume first that $r=1$. Write

$$
u=\prod_{n \in \mathbb{Z}} \beta_{n}^{j_{n}} \quad \text { and } \quad v=u^{-1} \varphi_{\left(i_{n}\right)_{n},(0)_{n}, 1}^{-1} .
$$

Let $\psi$ be the endomorphism of $\mathscr{L}_{k}$ defined by $\alpha \psi=\alpha \varphi_{\left(i_{n}\right)_{n},(0)_{n}, 1}^{-1}$ and $\xi \psi=v \xi$. In view of (5.2), we get

$$
\alpha \varphi_{\left(i_{n}\right)_{n},\left(j_{n}\right)_{n}, 1} \psi=\alpha \varphi_{\left(i_{n}\right)_{n},(0)_{n}, 1} \psi=\alpha
$$

and

$$
\xi \varphi_{\left(i_{n}\right)_{n},\left(j_{n}\right)_{n}, 1} \psi=(u \xi) \psi=\left(u \varphi_{\left(i_{n}\right)_{n},(0)_{n}, 1}^{-1}\right)(v \xi)=\xi
$$

hence $\varphi_{\left(i_{n}\right)_{n},\left(j_{n}\right)_{n}, 1} \psi=1$. Similarly, we can show that $\psi \varphi_{\left(i_{n}\right)_{n},\left(j_{n}\right)_{n}, 1}=1$; hence $\varphi\left(i_{n}\right)_{n},\left(j_{n}\right)_{n}, 1 \in \operatorname{Aut}\left(\mathscr{L}_{k}\right)$.

Assume now that $r=-1$. We have $\alpha \zeta \varphi_{\left(i_{n}\right)_{n},\left(j_{n}\right)_{n},-1}=\prod_{n \in \mathbb{Z}} \beta_{n}^{i_{n}}$ and

$$
\begin{aligned}
\xi \zeta \varphi_{\left(i_{n}\right)_{n},\left(j_{n}\right)_{n},-1} & =\xi^{-1} \varphi_{\left(i_{n}\right)_{n},\left(j_{n}\right)_{n},-1} \\
& =\xi \prod_{n \in \mathbb{Z}} \beta_{n}^{-j_{n}}=\left(\prod_{n \in \mathbb{Z}} \beta_{n+1}^{-j_{n}}\right) \xi=\left(\prod_{n \in \mathbb{Z}} \beta_{n}^{-j_{n-1}}\right) \xi .
\end{aligned}
$$

Thus $\zeta \varphi_{\left(i_{n}\right)_{n},\left(j_{n}\right)_{n},-1}=\varphi_{\left(i_{n}\right)_{n},\left(-j_{n-1}\right)_{n}, 1}$, which is an automorphism by the preceding case. Since $\zeta$ is an automorphism, so is $\varphi_{\left(i_{n}\right)_{n},\left(j_{n}\right)_{n},-1}$.

Lemma 5.6. Let $u, v \geq 2$ be coprime integers. Then $\mathscr{L}_{u} \cong\left\langle\alpha^{v}, \xi\right\rangle \leq \mathscr{L}_{u v}$.

Proof. By (5.1),

$$
\left\langle\alpha, \xi \mid \alpha^{u v},\left[\xi^{m} \alpha \xi^{-m}, \xi^{n} \alpha \xi^{-n}\right](m, n \in \mathbb{Z})\right\rangle
$$

is a presentation of $\mathscr{L}_{u v}$ and

$$
\left\langle\alpha, \xi \mid \alpha^{u},\left[\xi^{m} \alpha \xi^{-m}, \xi^{n} \alpha \xi^{-n}\right](m, n \in \mathbb{Z})\right\rangle
$$

is a presentation of $\mathscr{L}_{u}$. Let $H=\left\langle\alpha^{v}, \xi\right\rangle \leq \mathscr{L}_{u v}$. We claim that $H=\left\langle\alpha^{v}, \xi\right\rangle \leq \mathscr{L}_{u v}$ is isomorphic to $\mathscr{L}_{u}$.

Indeed, it follows from the given presentations that $\alpha \mapsto \alpha^{v}, \xi \mapsto \xi$ defines a surjective homomorphism $\theta$ from $\mathscr{L}_{u}$ onto $H$. Suppose that

$$
w=\left(\prod_{n \in \mathbb{Z}} \beta_{n}^{i_{n}}\right) \xi^{r} \in \operatorname{Ker} \theta,
$$

where $\left(i_{n}\right)_{n}$ is a function from $\mathbb{Z}$ into $\mathbb{Z}_{u}$ with finite support and $r \in \mathbb{Z}$. Then $1=w \theta=\left(\prod_{n \in \mathbb{Z}} \beta_{n}^{v i_{n}}\right) \xi^{r}$ yields $r=0$ and $u v \mid v i_{n}$ for every $n$. Thus $u \mid i_{n}$ for every $n$, and so $w=1$. Therefore, $\theta: \mathscr{L}_{u} \rightarrow H$ is an isomorphism. 
Lemma 5.7. Let $p_{1}, \ldots, p_{t}$ be distinct positive primes with $t \geq 2$. Let $c_{1} \geq 2$ and $c_{2}, \ldots, c_{t} \geq 1$. Then $\operatorname{Stab}_{p_{p_{1}^{c}}^{c}}(\xi)$ is a homomorphic image of $\operatorname{Stab}_{\mathcal{L}_{1}^{c}} c_{1} \ldots p_{t}^{c t}(\xi)$.

Proof. Write $u=p_{1}^{c_{1}}, v=p_{2}^{c_{2}} \ldots p_{t}^{c_{t}}$ and $k=u v$. Let $H=\left\langle\alpha^{v}, \xi\right\rangle \leq \mathscr{L}_{u v}$. By Lemma 5.6, we have $H \cong \mathscr{L}_{u}$.

We define

$$
\omega: \operatorname{Stab}_{\mathscr{L}_{k}}(\xi) \rightarrow \operatorname{Stab}_{H}(\xi),\left.\quad \psi \mapsto \psi\right|_{H} .
$$

We show that $H \psi \subseteq H$ for each $\psi \in \operatorname{Stab}_{\mathscr{L}_{k}}(\xi)$. Indeed, we may assume that $\alpha \psi=\prod_{n \in \mathbb{Z}} \beta_{n}^{i_{n}}$ for some function $\left(i_{n}\right)_{n}$ from $\mathbb{Z}$ into $\mathbb{Z}_{k}$ with finite support. Since $\xi \psi=\xi$ and $\alpha^{v} \psi=\prod_{n \in \mathbb{Z}} \beta_{n}^{v i_{n}}$, we get $H \psi \subseteq H$. This implies that $\omega$ is a (group) homomorphism.

We show next that $\omega$ is surjective. In view of Lemma 5.2, it suffices to show that

$$
\left\{\lambda, \eta_{j}, \delta_{m, p r} \mid j \in \mathbb{Z}_{k}^{*}, m \in \mathbb{Z} \backslash\{0\}, r \in \mathbb{Z}_{p^{s}}\right\} \subseteq \operatorname{Im} \omega
$$

(after translation from $\mathscr{L}_{u}$ to $H$ ).

Clearly, $\lambda$ in $H$ (defined by $\alpha^{v} \mapsto \xi \alpha^{v} \xi^{-1}$ and $\xi \mapsto \xi$ ) is the restriction of $\lambda$ from $\mathscr{L}_{k}$.

Consider next $\eta_{j}$ in $H$ (defined by $\alpha^{v} \mapsto\left(\alpha^{v}\right)^{j}$ and $\xi \mapsto \xi$, where $j \in \mathbb{Z}_{u}^{*}$ ). We can represent $j$ as an integer not divisible by $p_{1}$. Let $v^{\prime}$ be the product of the primes $p_{2}, \ldots, p_{t}$ which do not divide $j$, and set $j^{\prime}=j+p_{1}^{c_{1}} v^{\prime}$.

Since $j \in \mathbb{Z}_{u}^{*}, p_{1}$ does not divide $j$, and so it does not divide $j^{\prime}$. Suppose that $p_{i}$ divides $j^{\prime}$ for some $2 \leq i \leq t$. If $p_{i}$ does not divide $j$, then it divides $v^{\prime}$, and so it divides $j=j^{\prime}-p_{1}^{c_{1}} v^{\prime}$, a contradiction. If $p_{i}$ divides $j$, then it divides $p_{1}^{c_{1}} v^{\prime}=j^{\prime}-j$, and so must be a factor of $v^{\prime}$, also a contradiction.

Thus we can consider $j^{\prime} \in \mathbb{Z}_{k}^{*}$ and $\eta_{j^{\prime}} \in \operatorname{Stab} \mathscr{L}_{k}(\xi)$. We claim that $\eta_{j^{\prime}} \omega=\eta_{j}$. Since $\xi \eta_{j^{\prime}}=\xi=\xi \eta_{j}$, it remains to be shown that $\alpha^{v} \eta_{j^{\prime}}=\alpha^{v} \eta_{j}$. This is equivalent to show that $\alpha^{v j^{\prime}}=\alpha^{v j}$ in $\mathscr{L}_{k}$, i.e. $k$ divides $v j^{\prime}-v j$, i.e. $p_{1}^{c_{1}}$ divides $j^{\prime}-j$, which is clearly true.

Before dealing with the remaining case, we remark that the following generalization of (5.14) holds with the same proof:

$$
\text { if } p=p_{1} \ldots p_{t} \text { and } c=\max \left\{c_{1}, \ldots, c_{t}\right\} \text {, then } \delta_{m, p r}^{p^{c}}=1 \text {. }
$$

Indeed, we adapt $\Phi_{m}$ and $\Lambda$ in the obvious way, and everything works the same.

Now consider $\delta_{m, p_{1} r}$ in $H$ (defined by $\alpha^{v} \mapsto \alpha^{v} \beta_{m}^{v p_{1} r}$ and $\xi \mapsto \xi$ ). Since $p_{1}^{c^{1}-1}$ and $p_{2} \ldots p_{t}$ are coprime, there exist $r^{\prime}, y \in \mathbb{Z}$ such that $p_{2} \ldots p_{t} r^{\prime}+p_{1}^{c_{1}-1} y=r$. Writing $p=p_{1} \ldots p_{t}$, we consider now $\delta_{m, p r^{\prime}}$, which is in $\operatorname{Stab}_{\mathscr{L}_{k}}(\xi)$ by (5.18). 
We claim that $\delta_{m, p r^{\prime}} \omega=\delta_{m, p_{1} r}$. Since $\xi \delta_{m, p r^{\prime}}=\xi=\xi \delta_{m, p_{1} r}$, it remains to be shown that $\alpha^{v} \delta_{m, p r^{\prime}}=\alpha^{v} \delta_{m, p_{1} r}$. This is equivalent to showing that

$$
\alpha^{v} \beta_{m}^{v p r^{\prime}}=\alpha^{v} \beta_{m}^{v p_{1} r} \text { in } \mathscr{L}_{k} .
$$

So it suffices to show that $k$ divides $v p r^{\prime}-v p_{1} r$, i.e. $p_{1}^{c_{1}-1}$ divides $p_{2} \ldots p_{t} r^{\prime}-r$, which is certainly true. Therefore, $\omega$ is surjective as claimed.

Lemma 5.8. Let $k=p_{1} \ldots p_{s}$, where $p_{1}, \ldots, p_{s}$ are distinct positive primes and $s \geq 2$. For $\ell=1, \ldots, s$, let $\rho_{\ell}$ be the endomorphism of $\mathscr{L}_{k}$ with $\alpha \rho_{\ell}=\alpha^{p_{\ell}} \beta_{1}^{k / p_{\ell}}$ and $\xi \rho_{\ell}=\xi$. Then

(i) $\operatorname{Stab}_{\mathscr{L}_{k}}(\xi)=\left\langle\eta_{j}, \rho_{\ell} \mid j \in \mathbb{Z}_{k}^{*}, \ell \in\{1, \ldots, s\}\right\rangle$;

(ii) $\left\langle\lambda, \eta_{j}, \rho_{\ell} \mid j \in \mathbb{Z}_{k}^{*}, \ell \in\{1, \ldots, s-1\}\right\rangle$ is a finite index subgroup of $\operatorname{Stab}_{\mathscr{L}_{k}}(\xi)$.

Proof. (i) Let $\ell \in\{1, \ldots, s\}$. We must show that $\rho_{\ell}$ is an automorphism.

Write $k_{\ell}=\frac{k}{p_{\ell}}$. Since $p_{\ell}^{2}$ and $k_{\ell}^{2}$ are coprime, there exist $u_{\ell}, v_{\ell} \in \mathbb{Z}$ such that $p_{\ell}^{2} u_{\ell}+k_{\ell}^{2} v_{\ell}=1$. Let $\psi$ denote the endomorphism of $\mathscr{L}_{k}$ with $\alpha \psi=\alpha^{p_{\ell}} u_{\ell} \beta_{-1}^{k_{\ell} v_{\ell}}$ and $\xi \psi=\psi$. It follows from (5.5) that

$$
\rho_{\ell} \psi=\varphi_{\left(i_{0}^{\prime} i_{n}+i_{-1}^{\prime} i_{n+1}\right)_{n},(0)_{n}, 1} .
$$

- If $n=-1$, then $i_{0}^{\prime} i_{n}+i_{-1}^{\prime} i_{n+1}=k_{\ell} v_{\ell} p_{\ell}=0$ in $\mathbb{Z}_{k}$.

- If $n=0$, then $i_{0}^{\prime} i_{n}+i_{-1}^{\prime} i_{n+1}=p_{\ell} u_{\ell} p_{\ell}+k_{\ell} v_{\ell} k_{\ell}=1$.

- If $n=1$, then $i_{0}^{\prime} i_{n}+i_{-1}^{\prime} i_{n+1}=p_{\ell} u_{\ell} k_{\ell}=0$ in $\mathbb{Z}_{k}$.

Since $i_{0}^{\prime} i_{n}+i_{-1}^{\prime} i_{n+1}=0$ for all the remaining values of $n$, we get $\rho_{\ell} \psi=1$. In view of (5.4), $\rho_{\ell} \in \operatorname{Stab}_{\mathscr{L}_{k}}(\xi)$.

We show next that

$$
\alpha \rho_{\ell}^{m}=\alpha^{p_{\ell}^{m}} \beta_{m}^{k_{\ell}^{m}} \text { for every } m \geq 1 .
$$

We use induction on $m$. The case $m=1$ holds by definition. Assume that the claim holds for some $m \geq 1$. In view of (5.7), we get

$$
\alpha \rho_{\ell}^{m+1}=\alpha^{p_{\ell}^{m+1}} \beta_{m}^{k_{\ell}^{m}} p_{\ell} \beta_{1}^{p_{\ell}^{m} k_{\ell}} \beta_{m+1}^{k_{\ell}^{m+1}}=\alpha^{p_{\ell}^{m+1}} \beta_{m+1}^{k_{\ell}^{m+1}}
$$

where the last equality follows from the fact that $k \mid k_{\ell}^{m} p_{\ell}$ and $k \mid p_{\ell}^{m} k_{\ell}$, and so

$$
\beta_{m}^{k_{\ell}^{m}} p_{\ell} \beta_{1}^{p_{\ell}^{m} k_{\ell}}=1
$$

and (5.19) holds. 
Similarly, we can show that

$$
\alpha \rho_{\ell}^{-m}=\alpha^{p_{\ell}^{m} u_{\ell}^{m}} \beta_{-m}^{k_{\ell}^{m} v_{\ell}^{m}} \quad \text { for every } m \geq 1 .
$$

In view of (5.19), and since $k$ divides $k_{i} k_{j}$ whenever $i \neq j$, a straightforward induction shows that

$$
\alpha \rho_{1}^{m_{1}} \ldots \rho_{r}^{m_{r}}=\alpha^{p_{1}^{m_{1}} \ldots p_{r}^{m_{r}}} \prod_{i=1}^{r} \beta_{m_{i}}^{k_{i}^{m_{i}}} \prod_{\ell \in\{1, \ldots r\} \backslash\{i\}} p_{\ell}^{m_{\ell}}
$$

holds for all $r \in\{1, \ldots, s\}$ and $m_{1}, \ldots, m_{r}>0$.

Now let $\psi \in \operatorname{Stab}_{\mathscr{L}_{k}}(\xi)$. We prove that, for each $\ell \in\{1, \ldots, s\}$,

$$
\alpha^{k_{\ell}} \psi=\beta_{m_{\ell}}^{k_{\ell} j_{\ell}} \quad \text { for some } m_{\ell} \in \mathbb{Z} \text { and } j_{\ell} \in \mathbb{Z}_{\ell}^{*} \text {. }
$$

Indeed, if $H=\left\langle\alpha^{k_{\ell}}, \xi\right\rangle$, it follows from Lemma 5.6 that $H \cong \mathscr{L}_{p_{\ell}}$. Since it holds that $\alpha^{k_{\ell}} \psi=(\alpha \psi)^{k_{\ell}}$, we get $H \psi \subseteq H$. Also $H \psi^{-1} \subseteq H$ out of symmetry; hence $H=H \psi^{-1} \psi \subseteq H \psi \subseteq H$, yielding $H \psi=H$. Hence $\left.\psi\right|_{H} \in \operatorname{Stab}_{H}(\xi)$. Since $H \cong \mathscr{L}_{p_{\ell}}$, (5.22) follows from Lemma 5.1.

Now the greatest common divisor of $k_{1}, \ldots, k_{s}$ is obviously 1 , so there exist $x_{1}, \ldots, x_{s} \in \mathbb{Z}$ such that $k_{1} x_{1}+\cdots+k_{s} x_{s}=1$. It follows from (5.22) that

$$
\alpha \psi=\beta_{m_{1}}^{k_{1} j_{1} x_{1}} \ldots \beta_{m_{s}}^{k_{s} j_{s} x_{s}} .
$$

Note that the numbers $m_{\ell}$ are not necessarily nonzero. We show that, for every $\ell \in\{1, \ldots, s+1\}$,

$$
\begin{aligned}
\alpha \psi \rho_{s}^{-m_{s}} \ldots \rho_{\ell}^{-m_{\ell}}= & \beta_{m_{1}}^{k_{1} y_{1}} \ldots \beta_{m_{\ell-1}}^{k_{\ell-1} y_{\ell-1}} \alpha^{k_{\ell} y_{\ell}} \ldots \alpha^{k_{s} y_{s}} \\
& \text { for some } y_{1}, \ldots, y_{s} \in \mathbb{Z} .
\end{aligned}
$$

The formula for $\ell=s+1$ is to be interpreted as computing $\alpha \psi$, and so it is trivially true because of (5.23). Assume (5.24) holds for $\ell \in\{2, \ldots, s+1\}$. If $m_{\ell-1} \neq 0$, it follows from (5.19) or (5.20) that there exist some $z, w \in \mathbb{Z}$ such that

$$
\alpha \rho_{\ell-1}^{-m_{\ell-1}}=\alpha^{p_{\ell-1} z} \beta_{-m_{\ell-1}}^{k_{\ell-1} w} .
$$

This is in fact also true if $m_{\ell-1}=0$ because $p_{\ell-1}$ and $k_{\ell-1}$ are coprime. We apply now (5.7) to the automorphisms $\psi \rho_{s}^{-m_{s}} \ldots \rho_{\ell}^{-m_{\ell}}$ and $\rho_{\ell-1}^{-m_{\ell-1}}$ and using (5.24) and (5.25). Since $k=p_{\ell-1} k_{\ell-1}$ and it divides the product of any two distinct $k_{i}$, we get

$$
\begin{aligned}
\alpha \psi \rho_{s}^{-m_{s}} \ldots \rho_{\ell-1}^{-m_{\ell-1}}= & \beta_{m_{1}}^{k_{1} y_{1} p_{\ell-1} z} \ldots \beta_{m_{\ell-2}}^{k_{\ell-2} y_{\ell-2} p_{\ell-1} z} \\
& \cdot \alpha^{k_{\ell-1}^{2} y_{\ell-1} w} \alpha^{k_{\ell} y_{\ell} p_{\ell-1} z} \ldots \alpha^{k_{s} y_{s} p_{\ell-1} z},
\end{aligned}
$$


proving (5.24). In particular, for $\ell=1$, we get

$$
\alpha \psi \rho_{s}^{-m_{s}} \ldots \rho_{1}^{-m_{1}}=\alpha^{j}
$$

for some $j \in \mathbb{Z}_{k}$. Since automorphisms preserve order, we must have $j \in \mathbb{Z}_{k}^{*}$; hence $\alpha \psi \rho_{s}^{-m_{s}} \ldots \rho_{1}^{-m_{1}}=\alpha \eta_{j}$, yielding $\psi=\eta_{j} \rho_{1}^{m_{1}} \ldots \rho_{s}^{m_{s}}$, and we are done.

(ii) We show that

$$
\rho_{1}^{m} \ldots \rho_{s}^{m}=\lambda^{m}
$$

for $m=\left(p_{1}-1\right) \ldots\left(p_{s}-1\right)$.

Indeed, it follows from (5.21) that

$$
\alpha \rho_{1}^{m} \ldots \rho_{s}^{m_{s}}=\alpha^{k^{m}} \prod_{i=1}^{s} \beta_{m}^{k_{i}^{2 m}}=\beta_{m}^{\sum_{i=1}^{s} k_{i}^{2 m}} .
$$

Thus it suffices to show that $k$ divides $\sum_{i=1}^{s} k_{i}^{2 m}-1$. This is equivalent to having $p_{i}$ divide $k_{i}^{2 m}-1$ for $i=1, \ldots, s$. Since $p_{i}$ does not divide $k_{i}$, we have $k_{i}^{p_{i}-1} \equiv 1\left(\bmod p_{i}\right)$; hence $k_{i}^{2 m} \equiv 1\left(\bmod p_{i}\right)$. Therefore, $(5.26)$ holds.

Since $\operatorname{Stab}_{\mathscr{L}_{k}}(\xi)$ is abelian, it follows from part (i) and (5.26) that

$$
\operatorname{Stab}_{\mathscr{L}_{k}}(\xi)=\bigcup_{i=0}^{m-1}\left\langle\lambda, \eta_{j}, \rho_{\ell} \mid j \in \mathbb{Z}_{k}^{*}, \ell \in\{1, \ldots, s-1\}\right\rangle \rho_{s}^{i} .
$$

In view of Lemma 5.5, we can define $\Psi_{k}$ to be the set of all automorphisms of $\mathscr{L}_{k}$ of the form $\varphi_{\left(\varepsilon_{n-r}\right)_{n},\left(j_{n}\right)_{n}, 1}$, where $r \in \mathbb{Z}$ and $\left(j_{n}\right)_{n}$ is a function from $\mathbb{Z}$ to $\mathbb{Z}_{r}$.

Lemma 5.9. For every $k \geq 2, \Psi_{k} \unlhd \operatorname{Aut}\left(\mathscr{L}_{k}\right)$ and $\Psi_{k} \cong \mathscr{L}_{k}$.

Proof. Every element $x \in \mathscr{L}_{k}$ can be uniquely written in the form

$$
x=\xi^{r}\left(\prod_{n \in \mathbb{Z}} \beta_{n}^{j_{n}}\right),
$$

where $r \in \mathbb{Z}$ and $\left(j_{n}\right)_{n}$ is a function from $\mathbb{Z}$ to $\mathbb{Z}_{r}$.

Let $\mu: \mathscr{L}_{k} \rightarrow \Psi_{k}$ be defined by

$$
\left(\xi^{r}\left(\prod_{n \in \mathbb{Z}} \beta_{n}^{j_{n}}\right)\right) \mu=\varphi_{\left(\varepsilon_{n+r}\right)_{n},\left(j_{n}\right)_{n}, 1} .
$$

It is clear that $\mu$ is a bijection. 
Assume now that $x=\xi^{r}\left(\prod_{n \in \mathbb{Z}} \beta_{n}^{j_{n}}\right)$ and $x^{\prime}=\xi^{r^{\prime}}\left(\prod_{n \in \mathbb{Z}} \beta_{n}^{j_{n}^{\prime}}\right)$ are arbitrary elements of $\mathscr{L}_{k}$. We have

$$
\begin{aligned}
x x^{\prime} & =\xi^{r}\left(\prod_{n \in \mathbb{Z}} \beta_{n}^{j_{n}}\right) \xi^{r^{\prime}}\left(\prod_{n \in \mathbb{Z}} \beta_{n}^{j_{n}^{\prime}}\right) \\
& =\xi^{r+r^{\prime}}\left(\prod_{n \in \mathbb{Z}} \beta_{n-r^{\prime}}^{j_{n}}\right)\left(\prod_{n \in \mathbb{Z}} \beta_{n}^{j_{n}^{\prime}}\right)=\xi^{r+r^{\prime}}\left(\prod_{n \in \mathbb{Z}} \beta_{n}^{j_{n+r^{\prime}+j_{n}^{\prime}}}\right) ;
\end{aligned}
$$

hence

$$
\left(x x^{\prime}\right) \mu=\left(\xi^{r+r^{\prime}}\left(\prod_{n \in \mathbb{Z}} \beta_{n}^{j_{n+r^{\prime}+j_{n}^{\prime}}}\right)\right) \mu=\varphi_{\left(\varepsilon_{n+r+r^{\prime}}\right)_{n},\left(j_{n+r^{\prime}}+j_{n}^{\prime}\right)_{n}, 1} .
$$

On the other hand, using (5.2), we have

$$
\begin{aligned}
\alpha(x \mu)\left(x^{\prime} \mu\right) & =\alpha \varphi_{\left(\varepsilon_{n+r}\right)_{n},\left(j_{n}\right)_{n}, 1} \varphi_{\left(\varepsilon_{n+r^{\prime}}\right)_{n},\left(j_{n}^{\prime}\right)_{n}, 1}=\beta_{-r} \varphi_{\left(\varepsilon_{n+r^{\prime}}\right)_{n},\left(j_{n}^{\prime}\right)_{n}, 1} \\
& =\prod_{n \in \mathbb{Z}} \beta_{n}^{\varepsilon_{n+r^{\prime}+r}}=\alpha \varphi_{\left(\varepsilon_{n+r+r^{\prime}}\right)_{n},\left(j_{n+r^{\prime}}+j_{n}^{\prime}\right)_{n}, 1}=\alpha\left(\left(x x^{\prime}\right) \mu\right), \\
\xi(x \mu)\left(x^{\prime} \mu\right) & =\xi \varphi_{\left(\varepsilon_{n+r}\right)_{n},\left(j_{n}\right)_{n}, 1} \varphi_{\left(\varepsilon_{n+r^{\prime}}\right)_{n},\left(j_{n}^{\prime}\right)_{n}, 1} \\
& =\left(\left(\prod_{n \in \mathbb{Z}} \beta_{n}^{j_{n}}\right) \xi\right) \varphi_{\left(\varepsilon_{n+r^{\prime}}\right)_{n},\left(j_{n}^{\prime}\right)_{n}, 1} \\
& =\left(\prod_{n \in \mathbb{Z}} \prod_{m \in \mathbb{Z}} \beta_{m}^{\varepsilon_{m+r^{\prime}-n} j_{n}}\right)\left(\prod_{n \in \mathbb{Z}} \beta_{n}^{j_{n}^{\prime}}\right) \xi=\left(\prod_{n \in \mathbb{Z}} \beta_{n-r^{\prime}}^{j_{n}}\right)\left(\prod_{n \in \mathbb{Z}} \beta_{n}^{j_{n}^{\prime}}\right) \xi \\
& =\left(\prod_{n \in \mathbb{Z}} \beta_{n}^{j_{n+r^{\prime}}+j_{n}^{\prime}}\right) \xi=\xi \varphi_{\left(\varepsilon_{n+r+r^{\prime}}\right)_{n},\left(j_{\left.n+r^{\prime}+j_{n}^{\prime}\right)_{n}, 1}=\xi\left(\left(x x^{\prime}\right) \mu\right),\right.}
\end{aligned}
$$

and so $(x \mu)\left(x^{\prime} \mu\right)=\left(x x^{\prime}\right) \mu$. Therefore, $\mu$ is a group isomorphism and $\Psi_{k}$ is obviously a subgroup of $\operatorname{Aut}\left(\mathscr{L}_{k}\right)$.

It remains to show that $\Psi_{k}$ is normal. First we note that, for every $x \in \mathscr{L}_{k}$,

$x$ and $\alpha$ are conjugate if and only if $x=\beta_{r}$ for some $r \in \mathbb{Z}$.

Indeed, suppose that $x=y \alpha y^{-1}$ for some $y \in \mathscr{L}_{k}$. We may write $y=u \xi^{r}$ for some $u \in \operatorname{Fin}\left(\mathscr{L}_{k}\right)$ and $r \in \mathbb{Z}$. Since $\operatorname{Fin}\left(\mathscr{L}_{k}\right)$ is abelian, it follows that

$$
x=y \alpha y^{-1}=u \xi^{r} \alpha \xi^{-r} u^{-1}=\xi^{r} \alpha \xi^{-r}=\beta_{r}
$$

The converse implication holds trivially. Since $\alpha \varphi_{\left(\varepsilon_{n-r}\right)_{n},\left(j_{n}\right)_{n}, 1}=\beta_{r}$, it follows that

$$
\Psi_{k}=\left\{\psi \in \operatorname{Aut}_{+}\left(\mathscr{L}_{k}\right) \mid \alpha \psi \text { and } \alpha \text { are conjugate in } \mathscr{L}_{k}\right\}
$$


Let $\varphi \in \operatorname{Aut}\left(\mathscr{L}_{k}\right), \psi \in \Psi_{k}$. Clearly, $\varphi \psi \varphi^{-1} \in \operatorname{Aut}_{+}\left(\mathscr{L}_{k}\right)$. If $\psi=\varphi_{\left(\varepsilon_{n-r}\right)_{n},\left(j_{n}\right), 1}$, it follows from (5.2) that

$$
\beta_{m} \psi=\prod_{n \in \mathbb{Z}} \beta_{n}^{\varepsilon_{n-r-m}}=\beta_{r+m}=\xi^{r} \beta_{m} \xi^{-r}
$$

holds for every $m \in \mathbb{Z}$; hence $u \psi=\xi^{r} u \xi^{-r}$ for every $u \in \operatorname{Fin}\left(\mathscr{L}_{k}\right)$. In particular, for $u=\alpha \varphi$, we get

$$
\alpha \varphi \psi \varphi^{-1}=\left(\xi^{r} u \xi^{-r}\right) \varphi^{-1}=\left(\xi^{r} \varphi^{-1}\right) \alpha\left(\xi^{r} \varphi^{-1}\right)^{-1} .
$$

Thus $\varphi \psi \varphi^{-1} \in \Psi_{k}$ by (5.27), and so $\Psi_{k} \unlhd \operatorname{Aut}\left(\mathscr{L}_{k}\right)$.

We now aim at producing a generating set for $\operatorname{Aut}\left(\mathscr{L}_{k}\right)$. Let $\iota=\varphi_{\left(\varepsilon_{n}\right)_{n},\left(\varepsilon_{n}\right)_{n}, 1}$.

Lemma 5.10. For every $k \geq 2$,

(i) $\operatorname{Aut}_{+}\left(\mathscr{L}_{k}\right)=\left\langle\operatorname{Stab}_{\mathscr{L}_{k}}(\xi) \cup\{l\}\right\rangle$;

(ii) $\operatorname{Aut}\left(\mathscr{L}_{k}\right)=\left\langle\operatorname{Stab}_{\mathscr{L}_{k}}(\xi) \cup\{\iota, \zeta\}\right\rangle$.

Proof. (i) Write $S_{k}=\operatorname{Stab}_{\mathscr{L}_{k}}(\xi)$, and let

$$
\varphi=\varphi_{\left(i_{n}\right)_{n},\left(j_{n}\right)_{n}, 1} \in \operatorname{Aut}_{+}\left(\mathscr{L}_{k}\right) .
$$

By Lemma 5.9, we can take $\psi=\varphi_{\left(\varepsilon_{n}\right)_{n},\left(-j_{n}\right)_{n}, 1} \in \Psi_{k} \leq \operatorname{Aut}_{+}\left(\mathscr{L}_{k}\right)$. By (5.2), we have

$$
\begin{aligned}
\xi \varphi \psi & =\left(\left(\prod_{m \in \mathbb{Z}}\left(\beta_{m} \psi\right)^{j_{m}}\right) \xi\right) \psi=\left(\left(\prod_{m \in \mathbb{Z}} \beta_{m}^{j_{m}}\right) \xi\right) \psi \\
& =\left(\prod_{m \in \mathbb{Z}} \beta_{m}^{j_{m}}\right)\left(\prod_{m \in \mathbb{Z}} \beta_{m}^{-j_{m}}\right) \xi=\xi,
\end{aligned}
$$

and so we get $\varphi \psi \in S_{k}$; hence $\varphi \in \Psi_{k} S_{k}$. Since $\mathscr{L}_{k}=\langle\alpha, \xi\rangle$, it follows from Lemma 5.9 that

$$
\Psi_{k}=\langle\alpha \mu, \xi \mu\rangle=\langle\iota, \lambda\rangle .
$$

Since $\lambda \in S_{k}$, we get $\operatorname{Aut}_{+}\left(\mathscr{L}_{k}\right)=\left\langle S_{k} \cup\{\iota\}\right\rangle$.

(ii) follows from the fact that $\operatorname{Aut}_{+}\left(\mathscr{L}_{k}\right)$ is a subgroup of index 2 of $\operatorname{Aut}\left(\mathscr{L}_{k}\right)$ and $\zeta \in$ Aut_ $_{-}\left(\mathscr{L}_{k}\right)$.

Let FStab $\mathscr{L}_{k}(\xi)$ denote the set of all finite order automorphisms of $\mathscr{L}_{k}$ fixing $\xi$. Since $\operatorname{Stab}_{\mathscr{L}_{k}}(\xi)$ is abelian by (5.4), $\operatorname{FStab}_{\mathscr{L}_{k}}(\xi)$ is an abelian group itself.

We say that $k \geq 2$ is squarefree if there exists no prime $p \in \mathbb{N}$ such that $p^{2}$ divides $k$. 
Theorem 5.11. Let $k \geq 2$ have positive prime divisors $p_{1}, \ldots, p_{s}$. Then the following conditions are equivalent:

(i) $k$ is squarefree;

(ii) $\operatorname{Aut}\left(\mathscr{L}_{k}\right)$ is finitely generated;

(iii) Aut $_{+}\left(\mathscr{L}_{k}\right)$ is a finite extension of $\mathscr{L}_{k} \rtimes\left(\mathbb{Z}^{s-1} \times \mathbb{Z}_{k}^{*}\right)$.

If in addition $p_{i}$ divides $\left(\frac{k}{p_{i}}\right)^{2}-1$ for $i=1, \ldots, s$, then

$\operatorname{Aut}_{+}\left(\mathscr{L}_{k}\right) \cong \mathscr{L}_{k} \rtimes\left(\mathbb{Z}^{s-1} \times \mathbb{Z}_{k}^{*}\right) \quad$ and $\quad \operatorname{Aut}\left(\mathscr{L}_{k}\right) \cong\left(\mathscr{L}_{k} \rtimes\left(\mathbb{Z}^{s-1} \times \mathbb{Z}_{k}^{*}\right)\right) \rtimes C_{2}$.

Proof. (i) $\Rightarrow$ (iii). By Lemma 5.9, we have $\Psi_{k} \unlhd$ Aut $_{+}\left(\mathscr{L}_{k}\right)$. Suppose first that $s=1$ (so $k$ is prime). Define $A_{k}=\left\{\eta_{j} \mid j \in \mathbb{Z}_{k}^{*}\right\}$. It is straightforward to check that $\mathbb{Z}_{k}^{*} \rightarrow A_{k}, j \mapsto \eta_{j}$ is a group isomorphism (for arbitrary $k$, in fact). Moreover, $\Psi_{k} \cap A_{k}=\{\mathrm{id}\}$. Let $\varphi \in \operatorname{Aut}_{+}\left(\mathscr{L}_{k}\right)$. By Lemma 5.1, $\alpha \varphi=\beta_{r}^{j}$ for some $r \in \mathbb{Z}, j \in \mathbb{Z}_{k}^{*}$. Hence $\varphi \eta_{j-1} \in \Psi_{k}$, and $\operatorname{so} \varphi \in \Psi_{k} A_{k}$. Thus Aut $+\left(\mathscr{L}_{k}\right)=\Psi_{k} A_{k}$, and so

$$
\mathrm{Aut}_{+}\left(\mathscr{L}_{k}\right) \cong \Psi_{k} \rtimes A_{k} \cong \mathscr{L}_{k} \rtimes \mathbb{Z}_{k}^{*}
$$

by Lemma 5.9. Therefore, $\operatorname{Aut}\left(\mathscr{L}_{k}\right) \cong\left(\mathscr{L}_{k} \rtimes\left(\mathbb{Z}^{s-1} \times \mathbb{Z}_{k}^{*}\right)\right) \rtimes C_{2}$ by (5.17).

Assume now that $s \geq 2$. We define

$$
A_{k}=\left\langle\eta_{j}, \rho_{\ell} \mid j \in \mathbb{Z}_{k}^{*}, \ell \in\{1, \ldots, s-1\}\right\rangle .
$$

Since $\operatorname{Stab}_{\mathscr{L}_{k}}(\xi)$ is abelian by (5.4), Lemma 5.8 implies that every element of $\operatorname{Stab}_{\mathscr{L}_{k}}(\xi)$ can be written in the form $\rho_{1}^{a_{1}} \ldots \rho_{s}^{a_{s}} \eta_{j}$ for some $a_{1}, \ldots, a_{s} \in \mathbb{Z}$ and $j \in \mathbb{Z}_{k}^{*}$. We show that

$$
\rho_{1}^{a_{1}} \ldots \rho_{s}^{a_{s}} \eta_{j}=1 \quad \text { implies } \quad a_{1}=\cdots=a_{s}=0 \text { and } j=1 .
$$

Let

$$
I_{+}=\left\{i \in\{1, \ldots, r\} \mid a_{i}>0\right\}, \quad I_{-}=\left\{i \in\{1, \ldots, r\} \mid a_{i}<0\right\} .
$$

Suppose that $I_{+} \cup I_{-} \neq \emptyset$. Replacing by the inverses if needed, we may assume that $I_{+} \neq \emptyset$. Since $\operatorname{Stab}_{\mathscr{L}_{k}}(\xi)$ is abelian, $\rho_{1}^{a_{1}} \ldots \rho_{s}^{a_{s}} \eta_{j}=1$ implies

$$
\alpha \eta_{j} \prod_{i \in I_{-}} \rho_{i}^{-a_{i}}=\alpha \prod_{i \in I_{+}} \rho_{i}^{a_{i}} .
$$

It follows from (5.21) that

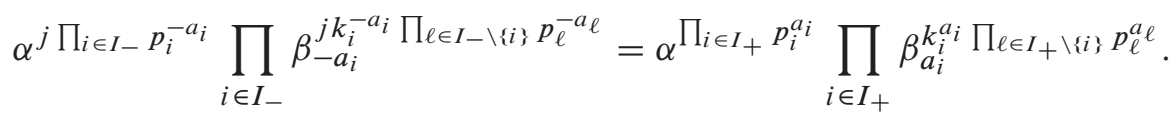


Comparing the powers of $\alpha$, we deduce that $k$ divides the difference

$$
j \prod_{i \in I_{-}} p_{i}^{-a_{i}}-\prod_{i \in I_{+}} p_{i}^{a_{i}}
$$

In particular, by taking $\ell \in I_{+}$, it follows that $p_{\ell}$ divides $j \prod_{i \in I_{-}} p_{i}^{-a_{i}}$, a contradiction. Thus $I_{+} \cup I_{-}=\emptyset$, i.e. $a_{1}=\cdots=a_{s}=0$. Now $j=1$ and (5.30) holds.

It follows from (5.30) that

$$
A_{k} \cong\left\langle\rho_{1}\right\rangle \times \cdots \times\left\langle\rho_{s-1}\right\rangle \times\left\{\eta_{j} \mid j \in \mathbb{Z}_{k}^{*}\right\} .
$$

We have already remarked that $\left\{\eta_{j} \mid j \in \mathbb{Z}_{k}^{*}\right\} \cong \mathbb{Z}_{k}^{*}$. Suppose that $\rho_{\ell}$ has finite order $m$. By (5.19), we get $\alpha p_{\ell}^{m}=\alpha$; hence $k$ (and therefore $p_{\ell}$ ) divides $p_{\ell}^{m}-1$, a contradiction. Thus each $\rho_{\ell}$ has infinite order, and so

$$
A_{k} \cong \mathbb{Z}^{s-1} \times \mathbb{Z}_{k}^{*}
$$

Consider the subgroup $\Psi_{k} A_{k}$ of $\operatorname{Aut}_{+}\left(\mathscr{L}_{k}\right)$. In view of Lemma 5.9, we have $\Psi_{k} A_{k}=A_{k} \Psi_{k}$. Suppose that $\psi \in A_{k} \cap \Psi_{k}$. Then $\psi=\varphi_{\left(\varepsilon_{n-r}\right)_{n},(0)_{n}, 1}=\lambda^{r}$ for some $r \in \mathbb{Z}$. Hence $\lambda^{r}=\rho_{1}^{a_{1}} \ldots \rho_{s-1}^{a_{s-1}} \eta_{j}$ for some $a_{1}, \ldots, a_{s-1} \in \mathbb{Z}$ and $j \in \mathbb{Z}_{k}^{*}$. Let $m=\left(p_{1}-1\right) \ldots\left(p_{s}-1\right)$. By (5.26), we have

$$
p_{1}^{m r} \ldots p_{s}^{m r}=\lambda^{m r}=\rho_{1}^{m a_{1}} \ldots \rho_{s-1}^{m a_{s-1}} \eta_{j}^{m},
$$

and so (5.30) yields $r=0$. Thus $A_{k} \cap \Psi_{k}=\{\operatorname{id}\}$. Since $\Psi_{k} \unlhd \Psi_{k} A_{k}$, we get, in view of Lemma 5.9 and (5.31),

$$
\Psi_{k} A_{k}=\left(\Psi_{k} \rtimes A_{k}\right) \cong\left(\mathscr{L}_{k} \rtimes\left(\mathbb{Z}^{s-1} \times \mathbb{Z}_{k}^{*}\right)\right) .
$$

Now we want to show that $\Psi_{k} A_{k}$ is a finite index subgroup of $\mathrm{Aut}_{+}\left(\mathscr{L}_{k}\right)$. Let $\varphi=\varphi_{\left(i_{n}\right)_{n},\left(j_{n}\right)_{n}, 1} \in \mathrm{Aut}_{+}\left(\mathscr{L}_{k}\right)$. Take $\psi=\varphi_{\left(\varepsilon_{n}\right)_{n},\left(-j_{n}\right)_{n}, 1} \in \Psi_{k}$. In view of (5.28) and (5.29), we have

$$
\alpha \varphi \psi=\prod_{n \in \mathbb{Z}} \beta_{n}^{i_{n}}, \quad \xi \varphi \psi=\left(\left(\prod_{n \in \mathbb{Z}} \beta_{n}^{j_{n}}\right) \xi\right) \psi=\xi ;
$$

hence $\varphi \psi \in \operatorname{Stab}_{\mathscr{L}_{k}}(\xi)$ and

$$
\operatorname{Aut}_{+}\left(\mathscr{L}_{k}\right)=\Psi_{k} \operatorname{Stab}_{\mathscr{L}_{k}}(\xi) .
$$

Since $\lambda \in \Psi_{k}$, it follows from Lemma 5.8 (ii) that $\Psi_{k} A_{k} \cap \operatorname{Stab}_{\mathscr{L}_{k}}(\xi)$ is a finite index subgroup of $\operatorname{Stab}_{\mathscr{L}_{k}}(\xi)$. Therefore, $\Psi_{k} A_{k}$ is a finite index subgroup of Aut $+\left(\mathscr{L}_{k}\right)$ and (iii) holds. 
Suppose now that $p_{i}$ divides $\left(\frac{k}{p_{i}}\right)^{2}-1$ for $i=1, \ldots, s$. Then it is straightforward to check that

$$
\lambda=\rho_{1} \ldots \rho_{s} .
$$

Indeed, in view of (5.21), we get

$$
\alpha \rho_{1} \ldots \rho_{s}=\alpha^{k} \beta_{1}^{\sum_{i=1}^{s} k_{i}^{2}}=\beta_{1}^{\sum_{i=1}^{s} k_{i}^{2}}
$$

hence it suffices to show that $k$ divides $\sum_{i=1}^{s} k_{i}^{2}-1$, which is equivalent to $p_{i}$ dividing $\left(\frac{k}{p_{i}}\right)^{2}-1$ for $i=1, \ldots, s$.

Now it follows from (5.34) that $\rho_{s} \in \Psi_{k} A_{k}$, yielding $\operatorname{Stab}_{\mathscr{L}_{k}}(\xi) \leq \Psi_{k} A_{k}$. By (5.33), we get Aut $+\left(\mathscr{L}_{k}\right)=\Psi_{k} A_{k}$, so Aut $+\left(\mathscr{L}_{k}\right) \cong \mathscr{L}_{k} \rtimes\left(\mathbb{Z}^{s-1} \times \mathbb{Z}_{k}^{*}\right)$ by $(5.32)$. Therefore, $\operatorname{Aut}\left(\mathscr{L}_{k}\right) \cong\left(\mathscr{L}_{k} \rtimes\left(\mathbb{Z}^{s-1} \times \mathbb{Z}_{k}^{*}\right)\right) \rtimes C_{2}$ by (5.17).

(iii) $\Rightarrow$ (ii). The groups $\mathscr{L}_{k}$ and $\mathbb{Z}^{s-1} \times \mathbb{Z}_{k}^{*}$ are finitely generated, so must be their semidirect product and all their finite extensions. Thus $\operatorname{Aut}_{+}\left(\mathscr{L}_{k}\right)$ is finitely generated and so is $\operatorname{Aut}\left(\mathscr{L}_{k}\right)$ by (5.17).

(ii) $\Rightarrow$ (i). Suppose now that $k$ is not squarefree. By Lemmas 5.3 and 5.7, $\operatorname{Stab}_{\mathscr{L}_{k}}(\xi)$ is not finitely generated. By Lemma 5.4, Aut $+\left(\mathscr{L}_{k}\right)$ is not finitely generated either. Since $\operatorname{Aut}_{+}\left(\mathscr{L}_{k}\right)$ is a finite index subgroup of $\operatorname{Aut}\left(\mathscr{L}_{k}\right)$, and every finite index subgroup of a finitely generated subgroup is finitely generated, it follows that $\mathrm{Aut}_{+}\left(\mathscr{L}_{k}\right)$ is not finitely generated.

\section{Uniform continuity}

Theorem 6.1. Every $\varphi \in \operatorname{Aut}\left(\mathscr{L}_{k}\right)$ is uniformly continuous with respect to the depth metric.

Proof. By Lemma 5.10, it is sufficient to show that every $\theta \in \operatorname{Stab}_{\mathscr{L}_{k}}(\xi)$ and that the maps $\iota$ and $\zeta$ are uniformly continuous with respect to the depth metric.

Assume first that $\theta \in \operatorname{Stab}_{\mathscr{L}_{k}}(\xi)$. By Proposition 4.3, it will suffice to show that

$$
\left(\operatorname{Stab}_{p}\left(\mathscr{L}_{k}\right)\right) \theta \subseteq \operatorname{Stab}_{p}\left(\mathscr{L}_{k}\right)
$$

holds for every $p \geq 0$.

Write $\alpha \theta=\beta_{i_{1}}^{r_{1}} \ldots \beta_{i_{m}}^{r_{m}}$. Then

$$
\beta_{\ell} \theta=\beta_{\ell+i_{1}}^{r_{1}} \ldots \beta_{\ell+i_{m}}^{r_{m}} \text { for every } \ell \in \mathbb{Z} .
$$

Assume that $\gamma \xi^{n} \in \operatorname{Stab}_{p}\left(\mathscr{L}_{k}\right)$ for some $\gamma \in \operatorname{Fin}\left(\mathscr{L}_{k}\right)$ and $n \in \mathbb{Z}$. Write

$$
\gamma=\beta_{i_{1}^{\prime}}^{r_{1}^{\prime}} \ldots \beta_{i_{s}^{\prime}}^{r_{s}^{\prime}}
$$


Let $q=p+1-\min \left\{i_{1}^{\prime}, \ldots, i_{s}^{\prime}\right\}$. Since $\operatorname{Stab}_{p}\left(\mathscr{L}_{k}\right) \unlhd \mathscr{L}_{k}$ and $\theta \in \operatorname{Aut}\left(\mathscr{L}_{k}\right)$, we have

$$
\begin{aligned}
\gamma \xi^{n} \in \operatorname{Stab}_{p}\left(\mathscr{L}_{k}\right) & \Longleftrightarrow\left(\xi^{q} \gamma \xi^{-q}\right) \xi^{n} \in \operatorname{Stab}_{p}\left(\mathscr{L}_{k}\right), \\
\left(\gamma \xi^{n}\right) \theta \in \operatorname{Stab}_{p}\left(\mathscr{L}_{k}\right) & \Longleftrightarrow\left(\left(\xi^{q} \gamma \xi^{-q}\right) \xi^{n}\right) \theta \in \operatorname{Stab}_{p}\left(\mathscr{L}_{k}\right) .
\end{aligned}
$$

Thus we may assume that $i_{v}^{\prime} \geq p+1$ for every $v$, replacing $\gamma \xi^{n}$ by $\left(\xi^{q} \gamma \xi^{-q}\right) \xi^{n}$ if needed.

Write

$$
f(t)=\sum_{v=1}^{s} r_{v}^{\prime}(1-t)^{i_{v}^{\prime}}, \quad g(t)=\sum_{u=1}^{m} r_{u}(1-t)^{i_{u}}, \quad h(t)=(1-t)^{-n} .
$$

Considering the formal series $X=\sum_{j=0}^{\infty} x_{j} t^{j}$, we have

$$
X \gamma \xi^{n}=(X+f(t)) h(t), \quad X\left(\left(\gamma \xi^{n}\right) \theta\right)=(X+f(t) g(t)) h(t) .
$$

Indeed, the first equality follows from the formal series interpretation of the $\beta_{i}$ and $\xi^{n}$. Since $\theta \in \operatorname{Stab}_{\mathscr{L}_{k}}(\xi)$, it suffices to show that $X(\gamma \theta)=X+f(t) g(t)$. This follows easily from the equality

$$
\gamma \theta=\left(\beta_{i_{1}^{\prime}}^{r_{1}^{\prime}} \ldots \beta_{i_{s}^{\prime}}^{r_{s}^{\prime}}\right) \theta=\prod_{u=1}^{m} \prod_{v=1}^{s} \beta_{i_{v}^{\prime}+i_{u}}^{r_{v}^{\prime} r_{u}} .
$$

Now let $W_{p+1}$ denote the principal ideal of $\mathbb{Z}_{k}[[t]]$ generated by $t^{p+1}$. Since $\gamma \xi^{n} \in \operatorname{Stab}_{p}\left(\mathscr{L}_{k}\right)$, it follows that $X \gamma \xi^{n}=x_{0}+x_{1} t^{1}+\ldots+x_{p} t^{p}+\cdots$, and so the first equation in (6.4) implies that

$$
(X+f(t)) h(t)-X=X \gamma \xi^{n}-X \in W_{p+1} \quad \text { for every } X \in \mathbb{Z}_{k}[[t]] .
$$

Considering the particular case $X=0$, we get $f(t) h(t) \in W_{p+1}$.

Using the second equation in (6.4), we want to show that

$$
X\left(\gamma \xi^{n}\right) \theta-X=(X+f(t) g(t)) h(t)-X \in W_{p+1} .
$$

Since

$$
(X+f(t) g(t)) h(t)-X=(X+f(t)) h(t)-X+f(t)(g(t)-1) h(t),
$$

the claim follows from $(X+f(t)) h(t)-X \in W_{p+1}$ and $f(t) h(t) \in W_{p+1}$. Since $X\left(\gamma \xi^{n}\right) \theta-X \in W_{p+1},\left(\gamma \xi^{n}\right) \theta \in \operatorname{Stab}_{p}\left(\mathscr{L}_{k}\right)$, and so (6.1) holds. Therefore, $\theta$ is uniformly continuous for every $\theta \in \operatorname{Stab}_{\mathscr{L}_{k}}(\xi)$. 
We show next that $\iota$ is uniformly continuous. By Proposition 4.3, it suffices to show that

$$
\left(\operatorname{Stab}_{p+1}\left(\mathscr{L}_{k}\right)\right) \iota \subseteq \operatorname{Stab}_{p}\left(\mathscr{L}_{k}\right)
$$

holds for every $p \geq 0$.

Assume that $\gamma \xi^{n} \in \operatorname{Stab}_{p+1}\left(\mathscr{L}_{k}\right)$ for some $\gamma \in \operatorname{Fin}\left(\mathscr{L}_{k}\right)$ and $n \in \mathbb{Z}$. Since

$$
\operatorname{Stab}_{p}\left(\mathscr{L}_{k}\right) \leq \mathscr{L}_{k} \quad \text { and } \quad \iota \in \operatorname{Aut}\left(\mathscr{L}_{k}\right),
$$

we have

$$
\begin{aligned}
\gamma \xi^{n} \in \operatorname{Stab}_{p}\left(\mathscr{L}_{k}\right) & \Longleftrightarrow \xi^{-n} \gamma^{-1} \in \operatorname{Stab}_{p}\left(\mathscr{L}_{k}\right), \\
\left(\gamma \xi^{n}\right) \iota \in \operatorname{Stab}_{p}\left(\mathscr{L}_{k}\right) & \Longleftrightarrow\left(\xi^{-n} \gamma^{-1}\right) \iota \in \operatorname{Stab}_{p}\left(\mathscr{L}_{k}\right) .
\end{aligned}
$$

Therefore, we may assume that $n \geq 0$, replacing $\gamma \xi^{n}$ by $\xi^{-n} \gamma^{-1}$ if needed.

Write $\gamma=\beta_{i_{1}}^{r_{1}} \ldots \beta_{i_{s}}^{r_{s}}$. Since $\iota \in \Psi_{k}$, (5.28) implies that $\beta_{\ell} \iota=\beta_{\ell}$ for every $\ell \in \mathbb{Z}$. Thus $\gamma \iota=\gamma$. On the other hand, a straightforward induction shows that

$$
\xi^{n} \iota=\beta_{0} \ldots \beta_{n-1} \xi^{n}
$$

for every $n \geq 0$. Write

$$
f(t)=\sum_{u=1}^{m} r_{u}(1-t)^{i_{u}}, \quad h(t)=(1-t)^{-n} .
$$

Considering the formal series $X=\sum_{j=0}^{\infty} x_{j} t^{j}$, we have

$$
X \gamma \xi^{n}=(X+f(t)) h(t), \quad X\left(\left(\gamma \xi^{n}\right) \iota\right)=\left(X+f(t)+\sum_{i=0}^{n-1}(1-t)^{i}\right) h(t)
$$

Indeed, the first equality follows from the formal series interpretation of the $\beta_{i}$ and $\xi^{n}$, and the second equality follows from $\gamma \iota=\gamma$ and (6.6).

Since $\gamma \xi^{n} \in \operatorname{Stab}_{p+1}\left(\mathscr{L}_{k}\right)$, we have $(X+f(t)) h(t)-X \in W_{p+2}$ for every $X \in \mathbb{Z}_{k}[[t]]$ by the first equality in (6.7). Hence

$$
X(h(t)-1)+f(t) h(t) \in W_{p+2} \quad \text { for every } X \in \mathbb{Z}_{k}[[t]] .
$$

Considering the particular case $X=0$, we get $f(t) h(t) \in W_{p+2}$ and consequently $X(h(t)-1) \in W_{p+2}$ for every $X \in \mathbb{Z}_{k}[[t]]$. Since $X$ is arbitrary, it follows that $h(t)-1 \in W_{p+2}$. But then

$$
\sum_{i=0}^{n-1}(1-t)^{i} h(t)=\frac{1-(1-t)^{n}}{1-(1-t)} h(t)=\frac{h(t)-1}{t} \in W_{p+1},
$$


yielding

$$
\begin{aligned}
X\left(\left(\gamma \xi^{n}\right) \theta\right)-X & =\left(X+f(t)+\sum_{i=0}^{n-1}(1-t)^{i}\right) h(t)-X \\
& =((X+f(t)) h(t)-X)+\sum_{i=0}^{n-1}(1-t)^{i} h(t) \in W_{p+1} .
\end{aligned}
$$

Hence $\left(\gamma \xi^{n}\right) \theta \in \operatorname{Stab}_{p}\left(\mathscr{L}_{k}\right)$, and so (6.5) holds. Therefore, $\iota$ is uniformly continuous.

Finally, we show that $\zeta$ is uniformly continuous. By Proposition 4.3, it will suffice to show that

$$
\left(\operatorname{Stab}_{p}\left(\mathscr{L}_{k}\right)\right) \theta \subseteq \operatorname{Stab}_{p}\left(\mathscr{L}_{k}\right)
$$

holds for every $p \geq 0$.

Recall that $\alpha \zeta=\alpha$ and $\xi \zeta=\xi^{-1}$ and so $\beta_{\ell} \zeta=\beta_{-\ell}$ for every $\ell \in \mathbb{Z}$. Assume now that $\gamma \xi^{n} \in \operatorname{Stab}_{p}\left(\mathscr{L}_{k}\right)$ for some $\gamma=\beta_{i_{1}}^{r_{1}} \ldots \beta_{i_{m}}^{r_{m}} \in \operatorname{Fin}\left(\mathscr{L}_{k}\right)$ and $n \in \mathbb{Z}$. Let $q=\max \left\{\left|i_{1}\right|, \ldots,\left|i_{m}\right|\right\}+1$. Then (6.2) and (6.3) (with $\theta$ replaced by $\zeta$ ) hold, so we can assume that $i_{1}, \ldots, i_{m}>0$ (replacing $\gamma \xi^{n}$ by $\left(\xi^{q} \gamma \xi^{-q}\right) \xi^{n}$ if needed).

Write

$$
f(t)=\sum_{u=1}^{m} r_{u}(1-t)^{i_{u}}, \quad g(t)=\sum_{u=1}^{m} r_{u}(1-t)^{-i_{u}}, \quad h(t)=(1-t)^{-n} .
$$

Recall that $h^{-1}(t)=(1-t)^{n}$. Considering the formal series $X=\sum_{j=0}^{\infty} x_{j} t^{j}$, we have

$$
X \gamma \xi^{n}=(X+f(t)) h(t), \quad X\left(\left(\gamma \xi^{n}\right) \zeta\right)=(X+g(t)) h^{-1}(t) .
$$

Indeed, the first equality follows from the formal series interpretation of the $\beta_{i}$ and $\xi^{n}$, and the second equality follows from $\gamma \zeta=\beta_{-i_{1}}^{r_{1}} \ldots \beta_{-i_{m}}^{r_{m}}$ and $\xi \zeta=\xi^{-1}$ and the same argument used to get the first equality.

Since $\gamma \xi^{n} \in \operatorname{Stab}_{p}\left(\mathscr{L}_{k}\right)$, we have

$$
(X+f(t)) h(t)-X \in W_{p+1} \quad \text { for every } X \in \mathbb{Z}_{k}[[t]]
$$

by (6.9). Considering the particular case $X=0$, we get $f(t) h(t) \in W_{p+1}$ and consequently $f(t) \in W_{p+1}$ (since $h(t)=1+t h^{\prime}(t)$ for some $\left.h^{\prime}(t) \in \mathbb{Z}_{k}[[t]]\right)$. We shall prove that $g(t) \in W_{p+1}$.

The coefficient of $t^{\ell}$ in $f(t)$ is $(-1)^{\ell} \sum_{u=1}^{m} r_{u}\left(\begin{array}{c}i_{u} \\ \ell\end{array}\right)$. Since $f(t) \in W_{p+1}$, we get

$$
\sum_{u=1}^{m} r_{u}\left(\begin{array}{c}
i_{u} \\
\ell
\end{array}\right)=0 \text { for } \ell=0, \ldots, p .
$$


For $\ell=0$, this is equivalent to $\sum_{u=1}^{m} r_{u}=0$. Since $i_{u} \geq 1$, for every $s \geq-1$, we have $\left(\begin{array}{c}i_{u}+s \\ 0\end{array}\right)=1$, and so

$$
\sum_{u=1}^{m} r_{u}\left(\begin{array}{c}
i_{u}+s \\
0
\end{array}\right)=0
$$

We show next that

$$
\sum_{u=1}^{m} r_{u}\left(\begin{array}{c}
i_{u}+s \\
\ell
\end{array}\right)=0
$$

for all $\ell \in\{1, \ldots, p\}$ and $s \geq 0$. We use induction on $s$.

The case $s=0$ follows from (6.10); hence we assume that $s>0$ and (6.12) holds for $s-1$ and every $\ell \in\{1, \ldots, p\}$. Indeed, we may use the induction hypothesis and (6.11) (if $\ell=1$ ) to deduce

$$
\sum_{u=1}^{m} r_{u}\left(\begin{array}{c}
i_{u}+s \\
\ell
\end{array}\right)=\sum_{u=1}^{m} r_{u}\left(\begin{array}{c}
i_{u}+s-1 \\
\ell
\end{array}\right)+\sum_{u=1}^{m} r_{u}\left(\begin{array}{c}
i_{u}+s-1 \\
\ell-1
\end{array}\right)=0+0=0 .
$$

Thus (6.12) holds for every $s$.

We now compute the coefficient $c_{\ell}$ of $t^{\ell}$ in $(1-t)^{-i_{u}}=\left(1+t+t^{2}+\ldots\right)^{i_{u}}$. Then $c_{\ell}$ is the number of decompositions of the form $\ell=a_{1}+\cdots+a_{i_{u}}$ with $a_{1}, \ldots, a_{i_{u}} \in \mathbb{N}$, and therefore also the number of decompositions of the form $\ell+i_{u}=b_{1}+\cdots+b_{i_{u}}$ with $b_{1}, \ldots, b_{i_{u}} \geq 1$. This number is well known to be $\left(\begin{array}{c}i_{u}+\ell-1 \\ i_{u}-1\end{array}\right)=\left(\begin{array}{c}i_{u}+\ell-1 \\ \ell\end{array}\right)$ (we must choose $i_{u}+\ell-1$ intermediate positions in a sequence of $\ell+i_{u}$ ones to bound the summands). It follows that the coefficient of $t^{\ell}$ in $g(t)$ is $\sum_{u=1}^{m} r_{u}\left(\begin{array}{c}i_{u}+\ell-1 \\ \ell\end{array}\right)$. By (6.11) and (6.12), we get $\sum_{u=1}^{m} r_{u}\left(\begin{array}{c}i_{u}+\ell-1 \\ \ell\end{array}\right)=0$ for $\ell=0, \ldots, p$. Thus $g(t) \in W_{p+1}$ as claimed.

Now

$$
\begin{aligned}
(X+g(t)) h^{-1}(t)-X= & g(t) h^{-1}(t)-\left(X-X h^{-1}(t)\right) \\
= & g(t) h^{-1}(t)-(X h(t)-X) h^{-1}(t) \\
= & g(t) h^{-1}(t)-((X+f(t)) h(t)-X) h^{-1}(t) \\
& \quad+f(t) \in W_{p+1}
\end{aligned}
$$

since $W_{p+1}$ is an ideal of $\mathbb{Z}_{k}[[t]]$. By (6.9), we get $\left(\gamma \xi^{n}\right) \zeta \in \operatorname{Stab}_{p}\left(\mathscr{L}_{k}\right)$, and so (6.8) holds. Therefore, $\zeta$ is uniformly continuous.

Since $\iota^{k}=\iota, \zeta^{2}=\zeta$ and the composition of uniformly continuous functions is uniformly continuous, it follows from Lemma 5.10 (ii) that every $\varphi \in \operatorname{Aut}\left(\mathscr{L}_{k}\right)$ is uniformly continuous.

Theorem 6.1 and Lemma 4.1 together finish the proof of Theorem 1.2. 


\section{Questions and observations}

We finish this paper with some open questions and suggestions for further research. After Theorem 6.1, it is very natural to ask the following question.

Questions 7.1. Does every automorphism of $\mathscr{L}_{k}$ extend to a continuous automorphism of $\operatorname{Aut}\left(T_{k}\right)$ ?

Next we note that the fixed point subgroup of an automorphism of $\mathscr{L}_{2}$ need not be finitely generated, even if the automorphism is inner.

Example 7.2. There exists $\theta \in \operatorname{Inn}\left(\mathscr{L}_{2}\right)$ such that $\operatorname{Fix}(\theta)$ is not finitely generated.

Indeed, let $\theta \in \operatorname{Inn}\left(\mathscr{L}_{2}\right)$ be defined by $\psi \theta=\alpha \psi \alpha^{-1}\left(\psi \in \mathscr{L}_{2}\right)$. Since Fin $\left(\mathscr{L}_{2}\right)$ is abelian, we have $\gamma \theta=\alpha \gamma \alpha=\alpha^{2} \gamma=\gamma$ for every $\gamma \in \operatorname{Fin}\left(\mathscr{L}_{2}\right)$. On the other hand, given $n \in \mathbb{Z}$, we have $\xi^{n} \theta=\alpha \xi^{n} \alpha=\beta_{0} \beta_{n} \xi^{n}$; hence $\xi^{n} \in \operatorname{Fix}(\theta)$ if and only if $n=0$. It follows that $\operatorname{Fix}(\theta)=\operatorname{Fin}\left(\mathscr{L}_{2}\right)$, an infinite abelian torsion group, therefore non-finitely generated.

Questions 7.3. Let $G \leq \operatorname{Aut}\left(T_{A}\right)$ be an automaton group and $\bar{G}$ its closure with respect to the depth metric.

(i) What conditions ensure that an automorphism of an automaton group

$$
G \leq \operatorname{Aut}\left(T_{A}\right)
$$

extends to a continuous automorphism of $\operatorname{Aut}\left(T_{A}\right)$ ?

(ii) Is every fixed point of $\bar{\varphi}$ a limit point of fixed points of $\varphi$ ?

Acknowledgments. The authors would like to thank Manuel Delgado for providing support in some calculations with the GAP mathematical software and an anonymous referee for helpful comments and references which enriched the exposition.

\section{Bibliography}

[1] S. Bachmuth, G. Baumslag, J. Dyer and H. Y. Mochizuki, Automorphism groups of two generator metabelian groups, J. Lond. Math. Soc. (2) 36 (1987), no. 3, 393-406.

[2] L. Bartholdi, R. Grigorchuk and V. Nekrashevych, From fractal groups to fractal sets, in: Fractals in Graz 2001, Trends Math., Birkhäuser, Basel (2003), 25-118.

[3] L. Bartholdi, R. I. Grigorchuk and Z. Šunik, Branch groups, in: Handbook of Algebra. Vol. 3, Elsevier/North-Holland, Amsterdam (2003), 989-1112. 
[4] L. Bartholdi and S. N. Sidki, The automorphism tower of groups acting on rooted trees, Trans. Amer. Math. Soc. 358 (2006), no. 1, 329-358.

[5] L. Bartholdi and P. Silva, Groups defined by automata, preprint (2010), https://arxiv. org/abs/1012.1531v1; to appear in Handbook of Automata Theory, Chapter 24.

[6] L. Bartholdi and Z. Šuniḱ, Some solvable automaton groups, in: Topological and Asymptotic Aspects of Group Theory, Contemp. Math. 394, American Mathematical Society, Providence (2006), 11-29.

[7] A. M. Brunner and S. Sidki, On the automorphism group of the one-rooted binary tree, J. Algebra 195 (1997), no. 2, 465-486.

[8] A. M. Brunner and S. Sidki, The generation of $\operatorname{GL}(n, \mathbf{Z})$ by finite state automata, Internat. J. Algebra Comput. 8 (1998), no. 1, 127-139.

[9] R. I. Grigorchuk, Just infinite branch groups, in: New Horizons in Pro-p Groups, Progr. Math. 184, Birkhäuser, Boston (2000), 121-179.

[10] K. B. Krohn and J. L. Rhodes, Algebraic theory of machines, in: Proceedings of the Symposium on Mathematical Theory of Automata (New York 1962), Polytechnic Press, Brooklyn (1963), 341-384.

[11] Y. Lavreniuk and V. Nekrashevych, Rigidity of branch groups acting on rooted trees, Geom. Dedicata 89 (2002), 159-179.

[12] V. Nekrashevych, Self-Similar Groups, Math. Surveys Monogr. 117, American Mathematical Society, Providence, 2005.

[13] P. V. Silva and B. Steinberg, On a class of automata groups generalizing lamplighter groups, Internat. J. Algebra Comput. 15 (2005), no. 5-6, 1213-1234.

[14] M. Stein, J. Taback and P. Wong, Automorphisms of higher rank lamplighter groups, Internat. J. Algebra Comput. 25 (2015), no. 8, 1275-1299.

[15] M. Vorobets and Y. Vorobets, On a free group of transformations defined by an automaton, Geom. Dedicata 124 (2007), 237-249.

Received March 3, 2020; revised January 16, 2021.

\section{Author information}

Corresponding author:

Francesco Matucci, Dipartimento di Matematica e Applicazioni,

Università degli Studi di Milano-Bicocca,

Office 3043, Via Cozzi 55, 20125 Milano, Italy.

E-mail: francesco.matucci@unimib.it

Pedro V. Silva, Centro de Matemática, Faculdade de Ciências,

Universidade do Porto, R. Campo Alegre 687, 4169-007 Porto, Portugal.

E-mail: pvsilva@fc.up.pt 